\title{
叶绿素-a 降解产物的硝酸铊氧化反应及其二氢卟吩衍生物的合成
}

\author{
高 娜 ${ }^{a}$ 张善国 ${ }^{b}$ 王 振 ${ }^{b}$ 王进军*,,$b$ \\ ( ${ }^{a}$ 烟台大学文经学院食品与生物工程系 烟台 264005) \\ ( ${ }^{b}$ 烟台大学化学化工学院 烟台 264005)
}

\begin{abstract}
摘要 以焦脱镁叶绿酸-a 甲酯为起始原料, 通过亲电加成、空气氧化、羟醛缩合和开环重排等经典的化学反应, 对其 $\mathrm{N}^{21}-\mathrm{N}^{23}$ 轴向两端的取代基团进行化学修饰和结构转换, 并在四吡咯大环分子的周环上构建了新的碳碳双键; 选择硝酸 铊为氧化剂, 对不同叶绿素降解产物的烯键实施氧化, 再利用所形成的甲酰甲氧基的化学反应活性, 继续与芳醛进行 Aldol 和 Friedläender 缩合反应, 完成了一系列未见报道的叶绿素类二氢卟吩衍生物的合成, 其化学结构均经 UV-Vis、 ${ }^{1} \mathrm{H}$ NMR、IR 及元素分析予以证实.
\end{abstract}

关键词 叶绿素-a; 二氢卟吩; 化学修饰; 氧化反应; 合成

\section{Oxidation Reactions of Chlorophyll Degradation Products with Thal- lum Nitrate and Synthesis of Chlorophyllous Chlorins Derivatives}

\author{
Gao, $\mathrm{Na}^{a} \quad$ Zhang, Shanguo ${ }^{b} \quad$ Wang, Zhen $^{b} \quad$ Wang, Jinjun ${ }^{* a, b}$ \\ ( ${ }^{a}$ Department of Food \& Biological Engineering, Wenjing College, Yantai University, Yantai 264005) \\ ( ${ }^{b}$ College of Chemistry and Chemical Engineering, Yantai University, Yantai 264005)
}

\begin{abstract}
Pyropheophorbide-a methyl ester was used as a starting material, and the chemical modifications and structural transformations along the terminals of $\mathrm{N}^{21}-\mathrm{N}^{23}$ axis were carried out by classic chemical reaction, such as electrophilic addition, allomerization, Aldol condensation and ringopening rearrangement, to build new carbon-carbon double bonds on the periphery of the tetrapyrrole macrocycle. Thallum nitrate was chose as oxidizing agent to oxidize the olefinic bonds of different chloropyll degradation products, and Aldol and Friedläender reactions with aromatic aldehydes were performed making use of the chemical activities of the formed formymethyl groups. The synthesis of a series of unreported chlorins related to chlorophyll was accomplished and their chemical structures were characterized by elemental analysis, UV-Vis, IR and ${ }^{1} \mathrm{H}$ NMR spectra.
\end{abstract}

Keywords chlorophyll-a; chlorin; chemical modification; oxidization reaction; synthesis

氧化反应是叶绿素二氢卟吩周环上最重要的化学 反应之一，其特定的官能转化能力、灵活的区域选择范 围和广泛的应用性质已经成为人们关注的热点 ${ }^{[1]}$. 在这 些天然大分子的芳香性氮杂轮烯上，由于四吡咯大环 $\pi-$ 体系的电荷密度分布不尽相同, 基本碳架中的多处富电 子区域可以与不同的氧化试剂发生氧化反应, 其中包括 空气氧化和试剂氧化. 同时, 在特定的条件下, 周环上 所连带的诸如烯键和醛基等活性取代基团, 也可以通过 氧化反应而转化成其他结构 ${ }^{[2,3]}$. 因此, 选择正确的化学 试剂和作用条件, 对叶绿素碳架上的活性反应位点实施
氧化, 是合成叶绿素类二氢卟吩衍生物的有效切入途 径. 为了深化叶绿素降解产物的化学研究, 本文基于前 期的工作基础 ${ }^{[2 b, 4 ~ 6]}$, 从焦脱镁叶绿酸-a 甲酯(1, 简称 $\mathrm{MPPa}$ )开始, 经外接环和 3-位乙烯基的结构修饰转化成 不同的叶绿素降解产物, 利用硝酸铊对叶绿素类二氢卟 吩的特殊氧化作用, 对其周环上原有的乙烯基和新建碳 碳双键实施氧化和进一步的官能团转换, 合成出一系列 未见报道的新型叶绿素类二氢卟吩衍生物. 在碱性条件 下, 通过空气氧化可以将焦脱镁叶绿酸-a 甲酯(1), 氧化 成 $13^{2}$-氧代焦脱镁叶绿酸-a 甲酯 $(2)^{[1 \mathrm{~d}]}$, 并且很容易高产

*E-mail: wjj1955@163.com

Received January 21, 2015; revised March 22, 2015; published online April 13, 2015.

Project supported by the the national natural Science Foundations of China (No. 21272048) and the Project of Shandong Applied Reaearch Centre of Gold Nanotechnology (2011).

国家自然科学基金(No. 21272048)和山东省黄金工程技术研究中心(2011 年度)资助项目. 
率地转化成红紫素-18 甲酯(3), 选择硝酸铊(III)为氧化 剂对其 3-位乙烯基实施氧化, 首先给出 $61 \%$ 的 C(3)-二 甲氧乙基红紫素-18 甲酯(4), 再经甲酸水溶液处理, 分 离出 $73 \%$ 的红紫素-18 醛酯(5). 以肌氨酸为催化剂, $\mathrm{MPPa}(1)$ 在 $80{ }^{\circ} \mathrm{C}$ 的甲苯中与多聚甲醛缩合, 以 $34 \%$ 的 产率给出 $13^{2}$-亚甲基焦脱镁叶绿酸-a 甲酯(6); 尝试用硝 酸铊(III)对其进行氧化, 结果得到难于分离的混合产物 ${ }^{[7]}$. 考虑到多个双键参与氧化会导致反应结果混乱, 因 此, 先用 $30 \%$ 溴化氢乙酸溶液处理二氢卟吩二烯 6 , 使 其 3-位乙烯基顺利地转换成 1-羟乙基, 所生成的二氢卟 吩醇 $7(49 \%)$ 只保留五外接环上的端烯双键, 然后与硝 酸铊(III)进行氧化反应, 产物不经分离, 直接在 $98 \%$ 甲 酸中搅拌过夜, 一步完成外接环的缩醛水解和 3-位醇脱 水成烯, 以 $34 \%$ 的总收率得到 $13^{2}$-羟亚甲基焦脱镁叶绿 酸-a 甲酯(8) (Scheme 1).

红紫素-18 甲酯(3)在甲苯中与苄胺的回流反应, 以 59\%的产率得到外接环酐胺解的 $N$-苯甲基红紫素-18二 酰亚胺(9), 继续用硝酸铊(III)对其 3-位乙烯基进行氧化, 分离出 $71 \%$ 的二氢卟吩甲缩醛(10), 再于 $80 \%$ 的甲酸溶 液对 3-位二甲氧乙基进行水解, 则高产率地转化成红紫 素-18 二酰亚胺醛(11, 80\%) (Scheme 2).

利用硝酸铊对 MPPa (1) 实施氧化, 除了高产率地得
到焦脱镁叶绿酸甲缩醛 $(12,68 \%)$ 以外，还分离出微量的 12-二甲氧甲基二氢卟吩(13); 化合物 12 在甲酸溶液水 解成二氢卟吩醛 14 后, 在甲醇钠催化下与苯甲醛进行 经典的羟醛缩合反应，结果分离出 $30 \%$ 的 3a-甲酰基-3b苯基取代的焦脱镁叶绿酸衍生物 15; 如果对二氢卟吩 缩醛 12 实施空气氧化, 可以分离出 $22 \%$ 外接环的氧化 产物 16, 在隔绝空气的条件下与邻苯二胺缩合并酸性 水解, 不经分离, 直接在与邻氨基苯甲醛发生 Friedläender 反应，以 24\%的产率获得 3-位连有喹啉基 的红紫素-18 二酰亚胺衍生物 17 (Scheme 3).

\section{1 结果与讨论}

\section{1 叶绿素二氢卟吩色基中的多电子区域与其氧化反} 应活性

叶绿素降解产物的基本碳架是 4 个吡咯子环携连四 个次甲基组合而成，其中 1 个含氮五元杂环两个 $\beta$-碳间 的双键被还原而以氢化吡咯的形式存在 ( $D$-子环). 所有 子环的 $\beta$-位上分别连有烷基、乙烯基和各种含羰基结构, 并在 17-和 18-位上形成两个手性中心. 在叶绿素二氢卟 吩色基中存在着多种芳香性多氮杂轮烯结构，其广域的 $\pi$-电子体系也决定着二氢卟吩大环的基本化学性质. 由 于叶绿素二氢卟吩色基缺失相应的对称性, 因而其四吡
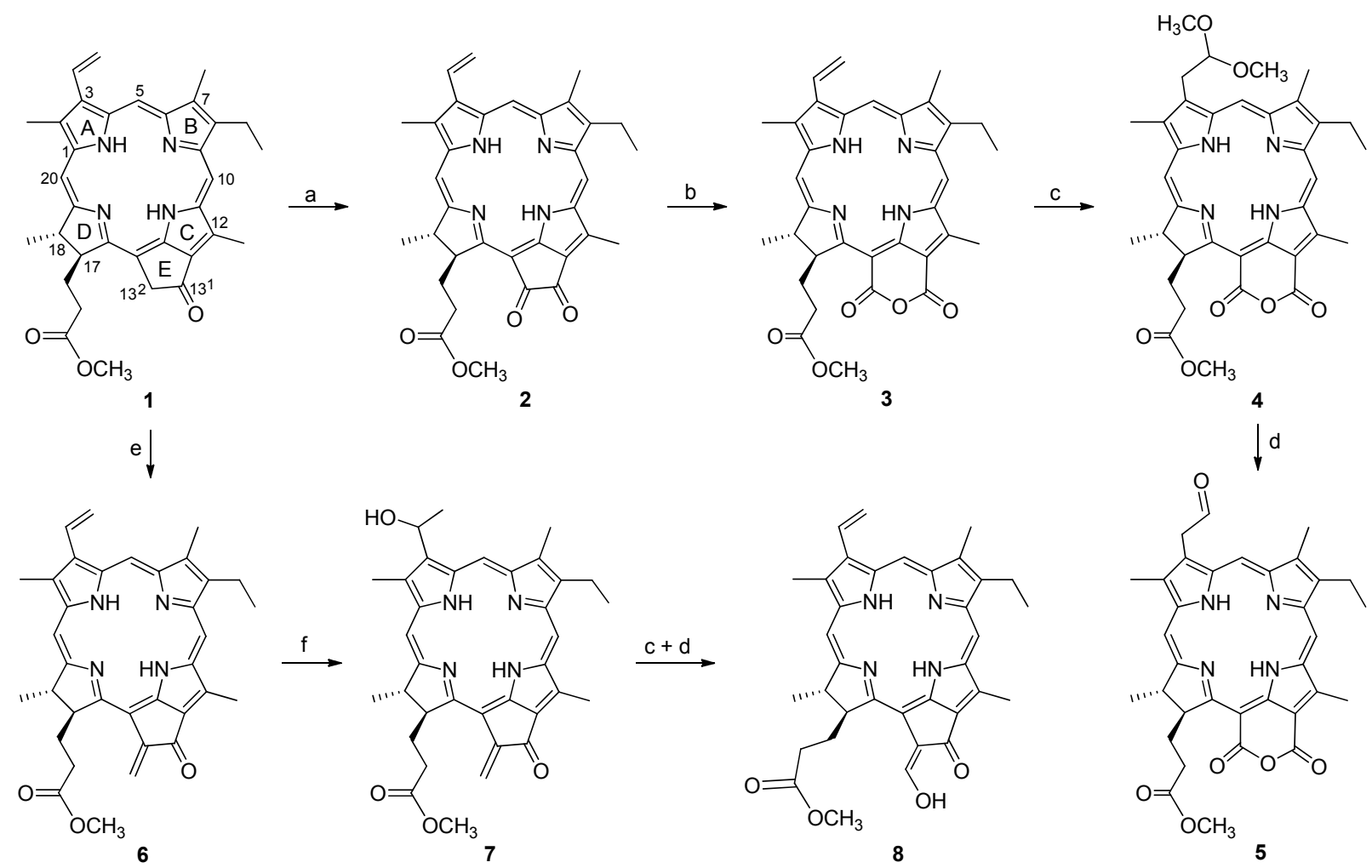

Reagents and conditions: (a) $\mathrm{LiOH} / \mathrm{THF} / \mathrm{H}_{2} \mathrm{O}$; (b) $\mathrm{NaOH} / \mathrm{MeOH}$; (c) Ti( $\left.\mathrm{NO}_{3}\right)_{3} / \mathrm{CH}_{2} \mathrm{Cl}_{2} ;$ (d) $80 \% \mathrm{HCO}_{2} \mathrm{H} ;(\mathrm{e})\left(\mathrm{CH}_{2} \mathrm{O}\right)_{n} / \mathrm{CH}_{3} \mathrm{NHCH} \mathrm{CO}_{2} \mathrm{H} / \mathrm{CH}_{3} \mathrm{Ph}$; (f) $\mathrm{HBr} / \mathrm{AcOH}$

图式 1 焦脱镁叶绿酸的结构修饰及其衍生物与硝酸铊的氧化反应

Scheme 1 The structural modification of pyropheophorbide and oxidation of its derivative with thallium nitrate 


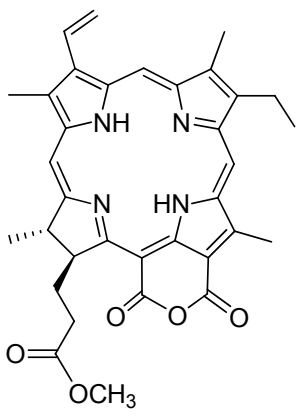

3
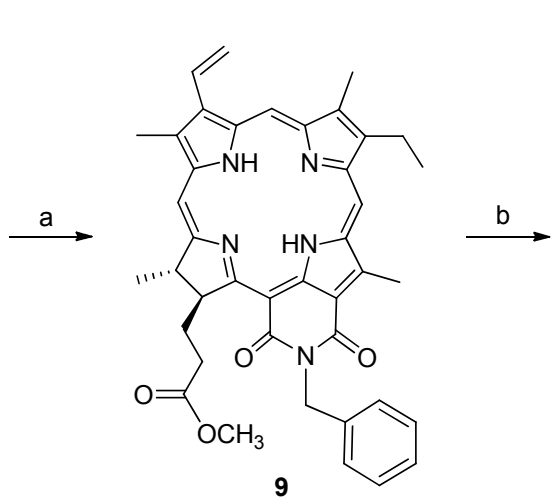

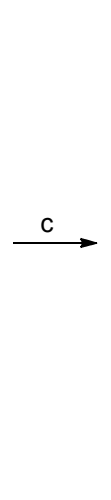

10

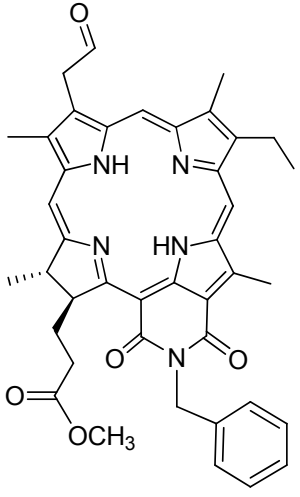

11

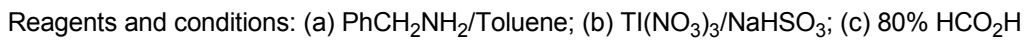

图式 2 红紫素-18 的酰基化及其二亚酰胺与硝酸铊的氧化反应

Scheme 2 The amidation of purpurin-18 and oxidation of its dimide with thallium nitrate
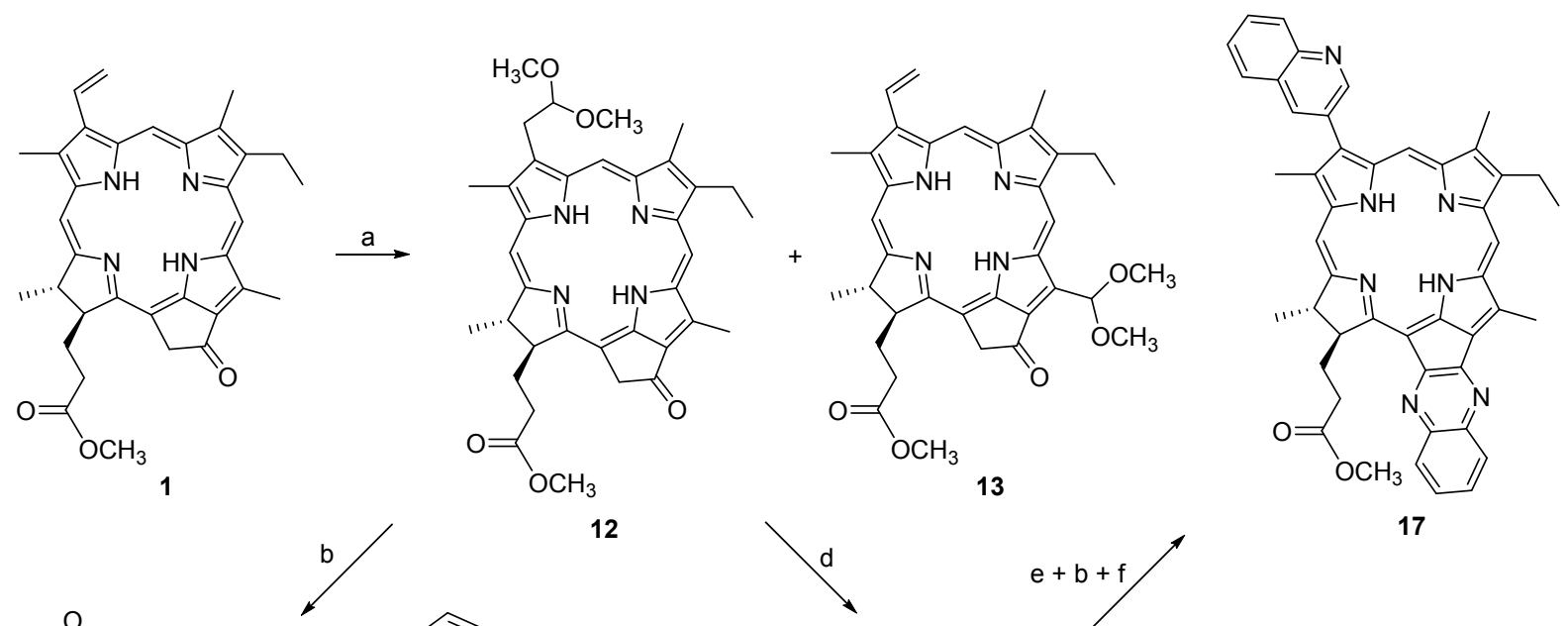

12
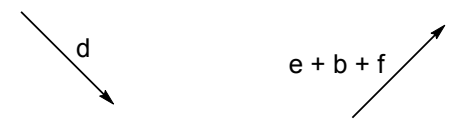

17

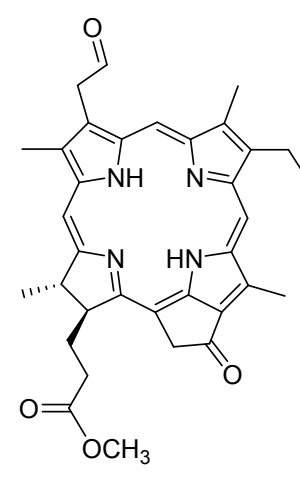

14
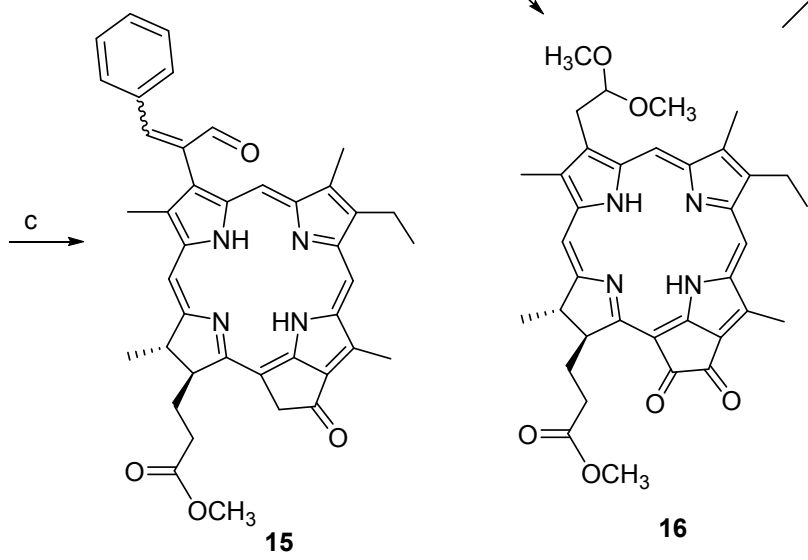

Reagents and conditions:

(a) $\mathrm{TI}\left(\mathrm{NO}_{3}\right)_{3} / \mathrm{NaHSO}_{3}$

(b) $80 \% \mathrm{HCO}_{2} \mathrm{H}$

(c) $\mathrm{PhCHO} / \mathrm{NaOMe} / \mathrm{MeOH}$

(d) $\mathrm{LiOH} / \mathrm{O}_{2} / \mathrm{MeOH} / \mathrm{THF}$

(e) $\mathrm{o}-\mathrm{Ph}\left(\mathrm{NH}_{2}\right)_{2} / \mathrm{Pyr} / \mathrm{TFA}$

(f) $\mathrm{o}-\mathrm{NH}_{2} \mathrm{PhCHO} / \mathrm{Pyr} / \mathrm{TFA}$

图式 3 焦脱镁叶绿酸与硝酸铊的氧化反应极其氧化产物的结构转换

Scheme 3 The oxidation of pyropheophorbide with thallium nitrate and the structural transformation of oxidized product

咯大环不能形成理想的共平面，贯通于二氢卟吩碳架的 离域 $\pi$ 键的电荷分配也不相同, 在色基的不同的位置上 能够形成多电子区域, 并在特定的反应条件下表现出良 好的给电子反应行为 ${ }^{[8]}$. 对于各种氧化剂而言, 氧化反 应的反应位点往往发生在电子云相对密集的活性区域. 组成大环色基的 3 个吡咯子环本身都包含 1 个 5 原子 6
电子的多电子次级离域体系, 因此叶绿素类二氢卟吩对 氧化剂的反应非常敏感. 例如，3-位的乙烯基的烯键多 电子性特征非常明显, 几乎可以进行所有碳碳双键的化 学反应, 我们曾用二氧化锰和间氯过氧化苯甲酸尝试对 C(3)-乙烯基实施氧化, 但均得到难于分离的复杂混合 产物 ${ }^{[7]}$; 如果选择相对温和的专用氧化剂, 可以完成 3- 


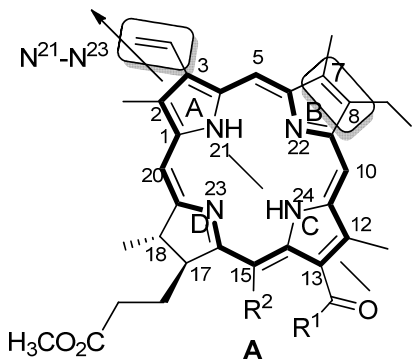

A
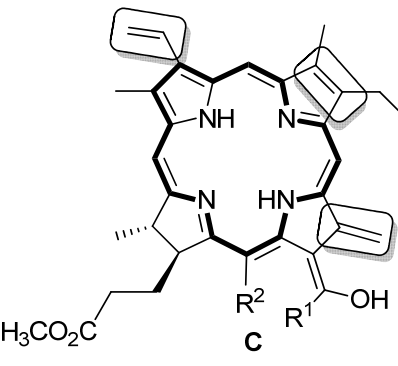

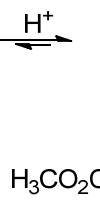

$\stackrel{\mathrm{H}^{+}}{\rightleftharpoons}$

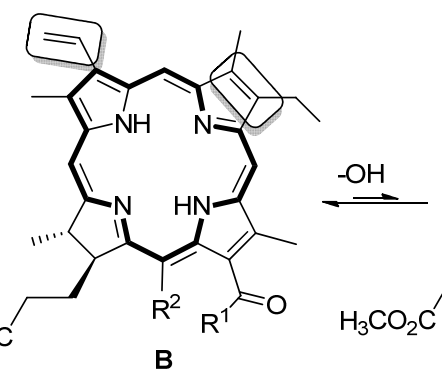

B

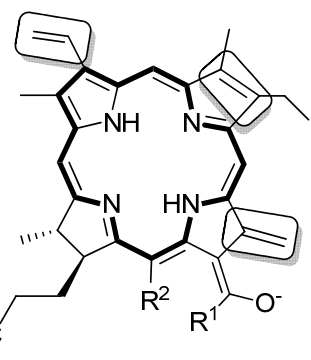

D

$\mathrm{R}^{1}+\mathrm{R}^{2}=$ exocyclic ring

图 1 叶绿素衍生物的芳香性共轭体系与其化学反应性

Figure 1 The aromatic conjugated system and their chemical reactivities of chlorophyll derivatives

位双键的氧化反应, 并可将其转化成各种含氧官能结 构 ${ }^{[2 b, 3 b]}$.

叶绿素降解产物的基本碳架可以形成不同的芳香 性多氮杂轮烯的 $\pi$ 电子离域体系. 图 1 中 $\mathbf{A}$ 和 $\mathbf{B}$ 是叶绿 素衍生物二氢卟吩色基中诸多氮杂轮烯中的两种代表 性的存在方式, 其中, 二氮杂-18-轮烯 $\mathbf{A}$ 是叶绿素大环 分子中具有最低能量的芳香性环系, 也是反映其化学行 为的最重要的依据结构. 在由 20 个 $\pi$ 电子参与的多层次 离域体系中(例如涵盖 $18 \pi$-电子的多氮杂轮烯、 $6 \pi$-电子 组成的吡咯子环和容纳 $5 \pi$-电子的含氮(富烯子环等), 7-8 位间的碳碳双键游离于最为稳定芳香性大环体系之外, 因此仍然保留着多电子的烯烃反应特征, 选择四氧化锇 对其实施氧化, 可以很容易地如期转化为邻位二醇结 构 ${ }^{[9]}$; 另一隐性碳碳双键来自于芳香性三氮杂-18-轮烯 B, 其 12-甲基通过与 13-位羰基的插烯作用, 在酸碱催 化下可以互变异构成烯醇结构 $\mathbf{C}$ 或者 $\mathbf{D}$, 在 12-碳和 12位甲基碳组成碳碳双键并瞬时游离于芳香性 $\pi$ 体系之 外, 因此, 12-位甲基能够形成类似于羰基 $\alpha$-位的化学反 应活性，同时也提供了一个隐性的多电子区域，例如， 在碱性空气氧化反应中，12-位甲基可以分别氧化成羟 甲基、醛基和羧酸酯基 ${ }^{[10]}$.

\section{2 叶绿素衍生物与硝酸铊的氧化作用以及产物与芳 醛的缩合反应}

根据叶绿素大环色基的结构变化可以确定，其 3-位 乙烯基、 $\mathrm{C}$ 环 $\beta$-位双键和 12-位甲基都能够体现出烯键 (见图 1 中框内结构)的化学反应特征, 同时, 利用叶绿 素周环上的活性基团进行相应的官能团转换, 又可以在 不同的位置上构建新的碳碳双键. 由于不同区域烯键的 氧化还原电位有所差异, 周边的立体环境也不尽相同, 所以, 确定氧化试剂和反应条件则是保证选择性氧化反 应的必要前提. 除此之外, 对特定的双键实施保护性结 构改造也是完成定向氧化的有效方法.

如图 2 所示: 当选用硝酸铊(III)作为氧化剂对叶绿
素降解产物 1 或者 3 的 C(3)-乙烯基实施氧化时, 三价铊 离子与 3-位双键首先发生亲电加成并形成鎓离子 $\mathbf{E}$, 随 后，甲醇作为亲核试剂从鎓离子三元环的反向完成负离 子加成, 中间铊化物 $\mathbf{F}$ 中不稳定的碳一铊键断裂后并带 走一对电子，离去一分子硝酸后其三价铊离子还原成一 价硝酸铊, 所生成的碳正离子随即发生重排生成更加稳 定的碳正离子 $\mathbf{G}$, 再与甲醇作用则得到氧化产物 4 或者 12; 3-位二甲氧乙基经酸处理以后很容易转化成 3-甲酰 甲基给出醛 $\mathrm{H}$ (例如化合物 5、11 和 14), 然后, 在碱性 条件下形成负碳离子 $\mathbf{I}$, 再分别与苯甲醛或者邻氨基苯 甲醛发生 Aldol 或者 Friedläender 缩合反应，分别得到芳 基化的二氢卟吩 15 和 17.

如果碳碳双键的 $\pi$ 键平面缺乏对称性, 硝酸铊(III) 的氧化将形成立体异构体而导致反应复杂化，例如，硝 酸铊(III)与具有双烯结构的二氢卟吩 $\mathbf{6}$ 的氧化反应就给 出难于分离的混合产物. 为此, 通过溴化氢的选择性亲 电加成反应, 先行将 3-位双键加以保护, 保留的外接环 端烯与硝酸铊(III)从二氢卟吩大环的上下两面形成两个 鎓离子 Ia 和 Ib, 再经过甲醇的反向亲核加成过程, 脱去 相应的硝酸和硝酸铊(I)分子而生成两个差向异构体 $\mathbf{J a}$ 和 $\mathbf{J b}$, 其 $13^{2}$-位的甲氧甲基再水解为 $13^{2}(R / S)$-非对映异 构体 $\mathbf{8}^{\prime}$, 然后, 异构成能够形成分子内氢键的更为稳定 的二氢卟吩 8 , 其 $13^{2}$-位的手性特征也随之消失. 化合 物 13 的形成也是经历了相似的氧化历程, 从焦脱镁叶 绿酸-a 甲酯的烯醇式异构体 1a 的 12-位端烯开始, 首先 形成两个差向异构的鎓离子 $\mathbf{K a}$ 和 $\mathbf{K b}$, 甲醇从大环上下 两面的亲核进攻在 12-位上两个不同取向的取代基团， 从而导致 12-位碳原子成为一个新的手性中心, 所生成 的氧化产物 13 '随即异构成二氢卟吩 13 , 其芳香性的二 氮杂-18-轮烯结构也得以恢复(图 3).

\section{2 结论}

通过焦脱镁叶绿酸外接环和 3-位乙烯基的结构修 

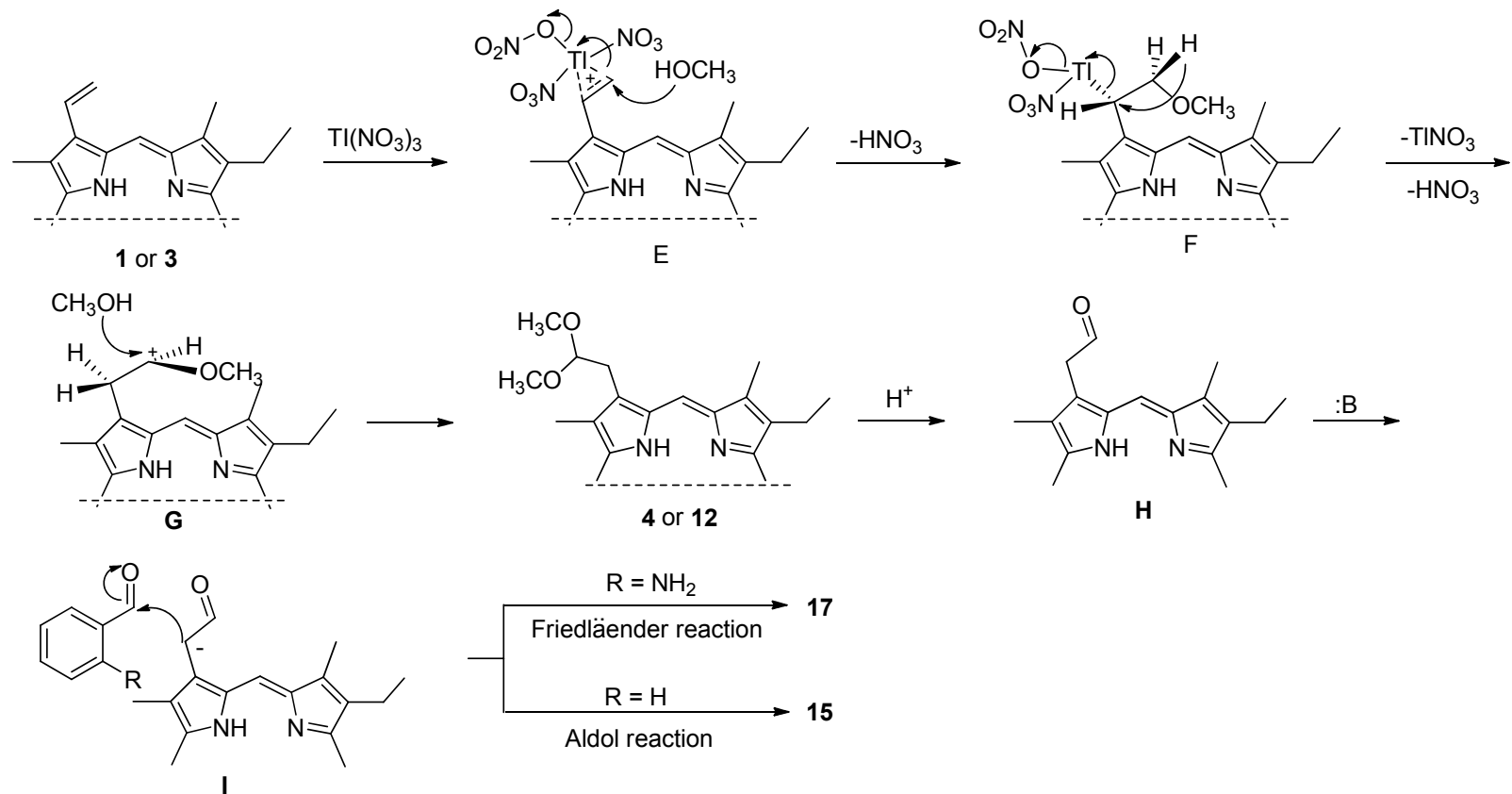

图 2 3-位乙烯基与硝酸铊的氧化及其与芳醛的缩合反应

Figure 2 The oxidation of C(3)-vinyl group with thaillum nitrate and the condensation with aromatic aldehyde

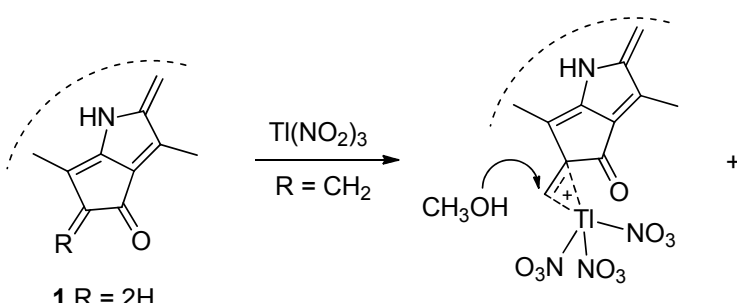

la

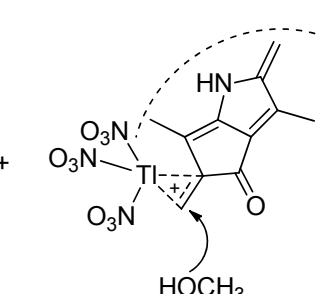

lb<smiles>COC(OC)[C@@]1(C)Nc2ccc(C)c(C)c2C1=O</smiles><smiles></smiles>

Jb $1 \mathrm{a}$

Ka

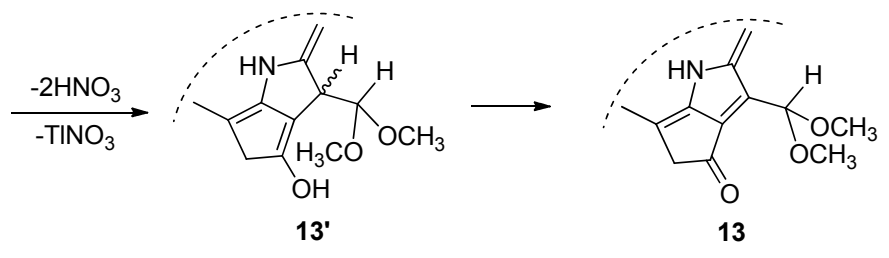

图 3 焦脱镁叶绿酸外接环的双键与硝酸铊的氧化反应

Figure 3 The oxidations of the double bonds in the exocyclic ring of pyropheophorbide with thaillum nitrate

饰，在叶绿素二氢卟吩碳架上构建出不同的活性反应区 域, 选择硝酸铊为氧化剂, 可以对其周环上不同形式的 碳碳双键实施有效的氧化, 进一步的官能团转换能够合
成出多种新型的叶绿素类二氢卟吩衍生物. 氧化反应是 叶绿素类二氢卟吩周环上最重要的化学反应之一, 选择 合适的氧化剂对环上富电子区域的氧化反应，具有理论 
研究和实际应用的双重意义, 利用叶绿素降解产物与硝 酸铊的氧化反应, 也是合成新型四吡咯大环化合物的有 效方法.

\section{3 实验部分}

\section{1 仪器与试剂}

元素分析用美国 Perkin-Elmer 2400 型元素分析仪 测定; IR 用美国 Perkin-Elmer 1730 型红外分光光度仪测 定 $(\mathrm{KBr}$ 压片); UV-Vis 用上海 UV-160A 型紫外分光光度 计测定; ${ }^{1} \mathrm{H}$ NMR 用德国 Brucker ARX-400 型核磁共振 仪测定, 内标为 TMS. 所用试剂均为分析纯或化学 纯. 焦脱镁叶绿酸-a 甲酯(1)按文献[11]制备; $13^{2}$-氧代焦 脱镁叶绿酸-a (2)按文献[1d]制备; 红紫素-18 甲酯(3)按 文献[7]制备.

\subsection{3-(2,2-二甲氧乙基)-3-去乙烯基红紫素-18 甲酯(4) 的合成}

在 $35 \mathrm{~mL}$ 甲醇中溶解 $116 \mathrm{mg}$ 化合物 2 (0.206 $\mathrm{mmol}$ ), 加入含有 $28 \mathrm{mg}$ 金属钠的 $6 \mathrm{~mL}$ 甲醇溶液; 室温 搅拌反应 $2 \mathrm{~h}$, 将反应混合物倒入 $20 \mathrm{~mL}$ 冷水中, 分出有 机层，水层用二氯甲烷萃取 $(15 \mathrm{~mL} \times 3)$, 合并有机相， 用无水硫酸钠干燥, 减压除尽溶剂. 将 $\mathbf{3}$ 的初产物溶解 于 $5 \mathrm{~mL}$ 二氯甲烷中, 再加 $20 \mathrm{~mL}$ 甲醇稀释, 冰浴下迅速 加入溶解于 $30 \mathrm{~mL}$ 甲醇的 $110 \mathrm{mg}$ 三水硝酸铊 $(0.247$ $\mathrm{mmol})$, 并继续摚拌反应 $45 \mathrm{~min}$, 然后加入 $30 \mathrm{~mL}$ 亚硫 酸氢钠甲醇饱和溶液, 室温下搅拌 $20 \mathrm{~min}$ 后, 滴加 2 $\mathrm{mL}$ 浓盐酸酸化反应液, 有大量白色沉淀生成, 除去所 生成的沉淀. 加水和二氯甲烷分层, 分出有机层并水洗 两次, 无水硫酸钠干燥, 减压浓缩, 剩余物经硅胶柱层 析分离 [洗脱剂: $V$ (石油醚) $: V$ (乙酸乙酯 $)=4: 1$ ], 得到 $81 \mathrm{mg}$ 黑绿色固体 4 (0.126 mmol), 产率 61\%. m.p. 229

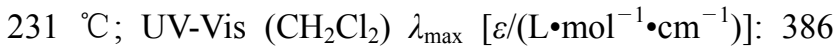
$\left(1.26 \times 10^{5}\right), \quad 409 \quad\left(9.32 \times 10^{4}\right), \quad 477 \quad\left(1.61 \times 10^{3}\right), \quad 545$ $\left(1.30 \times 10^{3}\right), 619\left(6.30 \times 10^{3}\right), 690\left(3.06 \times 10^{4}\right) ;{ }^{1} \mathrm{H}$ NMR $\left(\mathrm{CDCl}_{3}, 400 \mathrm{MHz}\right) \delta$ : -0.08 (br s, $\left.1 \mathrm{H}, \mathrm{NH}\right), 0.04$ (br s, $1 \mathrm{H}, \mathrm{NH}), 1.58$ (t, $\left.J=7.6 \mathrm{~Hz}, 3 \mathrm{H}, 8-\mathrm{CH}_{3}\right), 1.69$ (d, $J=7.2$ $\left.\mathrm{Hz}, 3 \mathrm{H}, 18-\mathrm{CH}_{3}\right), 2.38 \sim 2.46(\mathrm{~m}, 2 \mathrm{H}, 17 \mathrm{a}, 17 \mathrm{~b}-\mathrm{H}), 2.53 \sim$ $2.60(\mathrm{~m}, 2 \mathrm{H}, 17 \mathrm{a}+17 \mathrm{~b}-\mathrm{H}), 3.10,3.21,3.41,3.42,3.53$, 3.65 (each s, each $\left.3 \mathrm{H}, 18 \mathrm{H}, \mathrm{CH}_{3}+\mathrm{OCH}_{3}\right), 3.56(\mathrm{t}, J=7.6$ $\mathrm{Hz}, 2 \mathrm{H}, 8 \mathrm{a}-\mathrm{H}), 4.07$ (d, $J=5.4 \mathrm{~Hz}, 2 \mathrm{H}, 3 \mathrm{a}-\mathrm{H}), 4.32$ (q, $J=$ $7.2 \mathrm{~Hz}, 1 \mathrm{H}, 18-\mathrm{H}), 5.20$ (d, $J=8.3 \mathrm{~Hz}, 1 \mathrm{H}, 17-\mathrm{H}), 4.89$ (t, $J=5.4 \mathrm{~Hz}, 1 \mathrm{H}, 3 \mathrm{~b}-\mathrm{H}), \quad 8.46,9.24,9.45$ (each s, each $1 \mathrm{H}$, meso-H); IR (KBr) v: $3458(\mathrm{~N}-\mathrm{H}), 2959(\mathrm{C}-\mathrm{H}), 1737$, $1710(\mathrm{C}=\mathrm{O}), 1662(\mathrm{C}=\mathrm{C}), 1564$ (chlorin skeleton), 1443, 1241. 1172, $911 \mathrm{~cm}^{-1}$; EI-MS m/z: $641.3\left(\mathrm{M}+\mathrm{H}^{+}\right)$. Anal. calcd for $\mathrm{C}_{36} \mathrm{H}_{40} \mathrm{~N}_{4} \mathrm{O}_{7}$ : C 67.48, H 6.29, N 8.74; found C 67.30, H 6.21, N 8.90.

\subsection{3-甲酰甲基-3-去乙烯基红紫素-18 甲酯(5)的合成}

将 $98 \mathrm{mg}$ (0.153 mmol)化合物 4 溶解于 $10 \mathrm{~mL} \mathrm{88 \%}$ 的甲酸水溶液中, 室温搅拌 $24 \mathrm{~h}$, 加 $20 \mathrm{~mL}$ 水和 $40 \mathrm{~mL}$ 二氯甲烷萃取, 分出水层, 用 $15 \mathrm{~mL}$ 饱和碳酸氢钠溶液 和 $15 \mathrm{~mL}$ 水洗涤, 干燥浓缩后用硅胶柱层析分离[洗脱 剂: $V$ (石油醚) $: V$ (乙酸乙酯) $=4: 1$ ], 得 $66 \mathrm{mg}$ 黑色固 体 5 (0.112 mmol), 产率 73\%. m.p. 205 207 ${ }^{\circ} \mathrm{C}$; UV-Vis

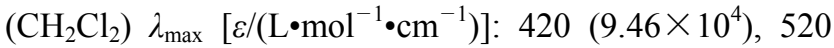
$\left(1.14 \times 10^{4}\right), \quad 5.67 \quad\left(4.80 \times 10^{3}\right), \quad 638 \quad\left(4.80 \times 10^{3}\right), \quad 672$ $\left(3.87 \times 10^{4}\right) \mathrm{nm} ;{ }^{1} \mathrm{H}$ NMR $\left(\mathrm{CDCl}_{3}, 400 \mathrm{MHz}\right) \delta:-2.41$ (br s, $1 \mathrm{H}, \mathrm{NH}), 0.58$ (br s, $1 \mathrm{H}, \mathrm{NH}), 1.73$ (t, $J=7.6 \mathrm{~Hz}, 1 \mathrm{H}$, $\left.8-\mathrm{CH}_{3}\right), 1.87\left(\mathrm{~d}, J=7.3 \mathrm{~Hz}, 3 \mathrm{H}, 18-\mathrm{CH}_{3}\right), 2.25 \sim 2.38(\mathrm{~m}$, $2 \mathrm{H}, 17 \mathrm{a}+17 \mathrm{~b}-\mathrm{H}), 2.63 \sim 2.81(\mathrm{~m}, 2 \mathrm{H}, 17 \mathrm{a}+17 \mathrm{~b}-\mathrm{H}), 3.35$, 3.47, 3.59, 3.80 (each s, each $3 \mathrm{H}, \mathrm{CH}_{3}+\mathrm{OCH}_{3}$ ), 3.75 (q, $J=7.6 \mathrm{~Hz} 2 \mathrm{H}, 8 \mathrm{a}-\mathrm{H}), 4.69$ (q, $J=7.3 \mathrm{~Hz}, 1 \mathrm{H}, 18-\mathrm{H}), 5.06$ (br s, 2H, 3a-H), 5.16 (d, $J=8.1 \mathrm{~Hz}, 1 \mathrm{H}, 17-\mathrm{H}), 9.01,9.58$, 9.81 (each s, each 1H, meso-H), 10.22 (s, 1H, CHO); IR (KBr) v: $3438(\mathrm{~N}-\mathrm{H}), 2958,2858(\mathrm{C}-\mathrm{H}), 1739,1701$ $(\mathrm{C}=\mathrm{O}), 1623(\mathrm{C}=\mathrm{N}), 1562$ (chlorin skeleton), 1463, 1371, 1228, 1168, 1029, 985, 910, $732 \mathrm{~cm}^{-1}$; EI-MS m/z: $754.6\left(\mathrm{M}+\mathrm{H}^{+}\right)$. Anal. calcd for $\mathrm{C}_{34} \mathrm{H}_{34} \mathrm{~N}_{4} \mathrm{O}_{6}: \mathrm{C}$ 68.67, $\mathrm{H}$ 5.76, N 9.42; found C 68.48, H 5.70, N 9.61.

\section{$3.413^{2}$-亚甲基焦脱镁叶绿酸-a 甲酯(6)的合成}

将 $267 \mathrm{mg}$ 焦脱镁叶绿酸-a 甲酯 $(\mathbf{1})(0.487 \mathrm{mmol})$ 、 $40 \mathrm{mg} N$-甲基甘氨酸和 $40 \mathrm{mg}$ 多聚甲醛溶解于 $40 \mathrm{~mL}$ 干 燥的甲苯中, 氮气保护下回流反应 $6 \mathrm{~h}$, 减压除去甲苯, 剩余物用硅胶柱层析分离[洗脱剂: $V$ (石油醚) : $V$ (乙酸 乙酯 $)=4: 1$ ], 得 $93 \mathrm{mg}$ 黄色固体 6 (0.166 mmol, 34\%). m.p. $188 \sim 191{ }^{\circ} \mathrm{C}$; UV-Vis $\left(\mathrm{CH}_{2} \mathrm{Cl}_{2}\right) \lambda_{\max }\left[\varepsilon /\left(\mathrm{L} \cdot \mathrm{mol}{ }^{-1} \bullet\right.\right.$ $\left.\mathrm{cm}^{-1}\right)$ ]: $412\left(1.87 \times 10^{4}\right), 431\left(1.60 \times 10^{4}\right), 523(1.87 \times$ $\left.10^{3}\right), 556\left(1.31 \times 10^{3}\right), 618\left(1.30 \times 10^{3}\right), 672\left(8.79 \times 10^{3}\right)$ $\mathrm{nm} ;{ }^{1} \mathrm{H} \mathrm{NMR}\left(\mathrm{CDCl}_{3}, 400 \mathrm{MHz}\right) \delta$ : -1.97 (br s, $\left.1 \mathrm{H}, \mathrm{NH}\right)$, -0.06 (br s, $1 \mathrm{H}, \mathrm{NH}), 1.68$ (t, $J=7.6 \mathrm{~Hz}, 3 \mathrm{H}, 8-\mathrm{CH}_{3}$ ), $1.86\left(\mathrm{~d}, J=7.4 \mathrm{~Hz}, 3 \mathrm{H}, 18-\mathrm{CH}_{3}\right), 1.98 \sim 2.10(\mathrm{~m}, 1 \mathrm{H}$, $17 \mathrm{a}+17 \mathrm{~b}-\mathrm{H}), 2.31 \sim 2.42(\mathrm{~m}, 1 \mathrm{H}, 17 \mathrm{a}+17 \mathrm{~b}-\mathrm{H}), 2.63 \sim$ $2.75(\mathrm{~m}, 2 \mathrm{H}, 17 \mathrm{a}+17 \mathrm{~b}-\mathrm{H}), 3.23,3.46,3.54,3.72$ (each s, each $3 \mathrm{H}, \mathrm{CH}_{3}+\mathrm{OCH}_{3}$ ), 3.68 (q, $\left.J=7.6 \mathrm{~Hz}, 2 \mathrm{H}, 8 \mathrm{a}-\mathrm{H}\right)$, 4.57 (q, $J=7.4 \mathrm{~Hz}, 1 \mathrm{H}, 18-\mathrm{H}), 4.62(\mathrm{~d}, J=9.2 \mathrm{~Hz}, 1 \mathrm{H}$, $17-\mathrm{H}), 6.19$ (dd, $J=11.6,1.1 \mathrm{~Hz}, 1 \mathrm{H}$, cis-3b-H), 6.34 (dd, $J=17.8,1.1 \mathrm{~Hz}, 1 \mathrm{H}$, trans $-3 \mathrm{~b}-\mathrm{H}), 6.51\left(\mathrm{~s}, 1 \mathrm{H}, 13^{2}=\mathrm{CH}\right)$, $6.83\left(\mathrm{~s}, 1 \mathrm{H}, 13^{2}=\mathrm{CH}\right), 8.00(\mathrm{dd}, J=17.8,11.6 \mathrm{~Hz}, 1 \mathrm{H}$, 3a-H), 8.70, 9.46, 9.57 (each s, each $1 \mathrm{H}$, meso-H); ${ }^{13} \mathrm{C}$ NMR (400 Hz, $\left.\mathrm{CDCl}_{3}, 400 \mathrm{MHz}\right) \delta: 10.98,11.28,12.20$, $17.01,17.49,19.47,19.47,23.54,29.30,31.23,49.79$, 
51.42, 51.69, 93.31, 97.04, 104.51, 106.04, 112.82, 128.46, $128.62,131.14,135.96,136.50,136.91,141.90,142.01$, $144.99,149.09,149.64,150.44,154.78,161.88,171.42$, 173.53, 187.13; IR (KBr) v: $3445(\mathrm{~N}-\mathrm{H}), 2958,2835$ $(\mathrm{C}-\mathrm{H}), 1736,1689(\mathrm{C}=\mathrm{O}), 1655(\mathrm{C}=\mathrm{C}), 1527$ (chlorin skeleton), 1400, 1286, 1189, $1094 \mathrm{~cm}^{-1}$; EI-MS m/z: 561.4 $\left(\mathrm{M}+\mathrm{H}^{+}\right)$. Anal. calcd for $\mathrm{C}_{35} \mathrm{H}_{36} \mathrm{~N}_{4} \mathrm{O}_{3}: \mathrm{C} 74.98, \mathrm{H} 6.47, \mathrm{~N}$ 9.99; found C 75.09, H 6.32, N 10.10.

\section{$3.53 \mathrm{a}(R / S)$-3-(1'-羟基乙基)-132-亚甲基-3-去乙烯基 焦脱镁叶绿酸-a 甲酯(7)的合成}

在 $25 \mathrm{~mL} \mathrm{30 \%}$ 的溴化氢乙酸溶液中溶解 $137 \mathrm{mg}$ 二 氢卟吩 6 (0.244 mmol), 室温摚拌反应 $16 \mathrm{~h}$, 将反应混合 物倒进 $120 \mathrm{~g}$ 碎冰中, 先后加入 $55 \mathrm{~mL}$ 二氯甲烷和 40 $\mathrm{mL}$ 水, 分出有机层, 水层用二氯甲烷萃取 $(25 \mathrm{~mL} \times 3)$, 合并有机层, 水洗后用无水硫酸钠干燥, 减压除去溶剂, 剩余物经硅胶柱层析 [洗脱剂: $V$ (石油醚) : $V$ (乙酸乙 酯 $)=5: 1$, 得 $69 \mathrm{mg}$ 黄色产物 7 (0.120 mmol, 49\%). m.p. $203 \sim 205{ }^{\circ} \mathrm{C}$; UV-Vis $\left(\mathrm{CH}_{2} \mathrm{Cl}_{2}\right) \lambda_{\max }\left[\varepsilon /\left(\mathrm{L} \cdot \mathrm{mol}^{-1} \bullet\right.\right.$ $\left.\mathrm{cm}^{-1}\right)$ ]: $408\left(1.12 \times 10^{5}\right), 428\left(1.18 \times 10^{4}\right), 554(1.09 \times$ $\left.10^{4}\right), 608\left(9.87 \times 10^{3}\right), 666\left(6.54 \times 10^{4}\right) \mathrm{nm} ;{ }^{1} \mathrm{H}$ NMR $\left(\mathrm{CDCl}_{3}, 400 \mathrm{MHz}\right) \delta$ : $-2.30(-2.25)($ br s, $1 \mathrm{H}, \mathrm{NH}),-$ 0.42 (br s, 1H, NH), 1.60 (1.59) (d, J=7.0 Hz, 3H, $\left.18-\mathrm{CH}_{3}\right), 1.75(1.76)\left(\mathrm{t}, J=7.6 \mathrm{~Hz}, 3 \mathrm{H}, 8-\mathrm{CH}_{3}\right), 1.84 \sim$ $1.97(\mathrm{~m}, 1 \mathrm{H}, 17 \mathrm{a}+17 \mathrm{~b}-\mathrm{H}), 2.09(\mathrm{~d}, J=6.4 \mathrm{~Hz}, 3 \mathrm{H}$, $\left.3 \mathrm{a}-\mathrm{CH}_{3}\right), 2.26 \sim 2.37(\mathrm{~m}, 1 \mathrm{H}, 17 \mathrm{a}+17 \mathrm{~b}-\mathrm{H}), 2.40 \sim 2.57$ (m, 2H, 17a+17b-H), 3.19 (3.20), 3.37 (3.39), 3.53, 3.66 (3.68) (each s, each $\left.3 \mathrm{H}, \mathrm{CH}_{3}+\mathrm{OCH}_{3}\right), 3.56$ (q, $J=7.6 \mathrm{~Hz}$, 2H, 8a-H), 4.48 (4.49) (q, $J=7.4 \mathrm{~Hz}, 1 \mathrm{H}, 18-\mathrm{H}), 4.54$ (d, $J$ $=10.0 \mathrm{~Hz}, 1 \mathrm{H}, 17-\mathrm{H}), 6.29(6.33)(\mathrm{t}, J=6.4 \mathrm{~Hz}, 1 \mathrm{H}$, $3 \mathrm{~b}-\mathrm{H}), 6.43$ (6.46) (s, 1H, 13 $\left.{ }^{2}=\mathrm{CH}\right), 6.76(6.78)(\mathrm{s}, 1 \mathrm{H}$, $13^{2}=\mathrm{CH}$ ), 8.59 (8.60), 9.27, 9.70 (9.66) (each s, each $1 \mathrm{H}$, meso-H); ${ }^{13} \mathrm{C} \mathrm{NMR}\left(400 \mathrm{~Hz}, \mathrm{CDCl}_{3}\right) \delta: 11.27,11.92$, 17.32, 17.59 (17.64), 19.38, 20.70, 29.89, 31.08 (31.18), 38.39 (38.54), 51.47 (51.57), 51.74 (51.82), 70.19 (70.29), 95.02 (95.05), 99.44 (99.91), 103.20, 106.19 (106.39), 129.85 (129.95), 131.76 (131.94), 133.27 (133.42), 136.98, 137.40 (137.57), 139.80, 144.84, 147.26 (147.44), 152.25 (152.32), 152.68 (152.74), $161.03 \quad$ (161.25), 170.87 (170.91), 173.38, 196.06 (196.11); IR (KBr) v: $3458(\mathrm{~N}-$ $\mathrm{H}), 2926(\mathrm{C}-\mathrm{H}), 1739,1701(\mathrm{C}=\mathrm{O}), 1616(\mathrm{C}=\mathrm{C}), 1550$ (chlorin skeleton), 1517, 1463, 1363, 1226, 1039, 738 $\mathrm{cm}^{-1}$; EI-MS $m / z$ : $579.4\left(\mathrm{M}+\mathrm{H}^{+}\right)$. Anal. calcd for: $\mathrm{C}_{35} \mathrm{H}_{38} \mathrm{~N}_{4} \mathrm{O}_{4} \mathrm{C}$ 72.64, $\mathrm{H}$ 6.62, N 9.68; found $\mathrm{C} 72.52, \mathrm{H}$ $6.56, \mathrm{~N} 9.88$.

\section{$3.613^{2}$-羟亚甲基焦脱镁叶绿酸-a 甲酯(8)的合成}

将 $137 \mathrm{mg}$ 二氢卟吩 $7(0.237 \mathrm{mmol})$ 溶解于 $5 \mathrm{~mL}$ 二 氯甲烷中, 再加 $20 \mathrm{~mL}$ 甲醇并冰浴冷却, 搅拌下一次性 加入含有 $150 \mathrm{mg}$ 三水硝酸铊的 $30 \mathrm{~mL}$ 甲醇, 并继续搅 拌反应 $45 \mathrm{~min}$, 然后加入 $30 \mathrm{~mL}$ 亚硫酸氢钠甲醇饱和溶 液, 室温下搅拌 $20 \mathrm{~min}$ 后, 滴加 $2 \mathrm{~mL}$ 浓盐酸并继续搅 拌反应 $1 \mathrm{~h}$, 过滤除去白色沉淀, 加入 $20 \mathrm{~mL}$ 水和 $40 \mathrm{~mL}$ 二氯甲烷分层, 分出有机层并水洗两次, 无水硫酸钠干 燥，减压浓缩，将浓缩物重新溶解于 $10 \mathrm{~mL} 80 \%$ 的甲酸 水溶液中, 室温搅拌 $24 \mathrm{~h}$, 加 $20 \mathrm{~mL}$ 水和 $40 \mathrm{~mL}$ 二氯甲 烷萃取, 分出水层, 先后用 $15 \mathrm{~mL}$ 饱和碳酸氢钠溶液和 $15 \mathrm{~mL}$ 水洗涤, 干燥浓缩后用硅胶柱层析分离[洗脱剂: $V$ (石油醚) $: V$ (乙酸乙酯 $)=4: 1]$, 得到 $46 \mathrm{mg}$ 黑绿色固 体 8 (0.081 mmol), 产率 34\%. m.p. 191 $194^{\circ} \mathrm{C}$; UV-Vis

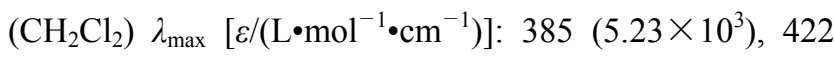
$\left(1.07 \times 10^{4}\right), \quad 512 \quad\left(9.42 \times 10^{2}\right), \quad 561 \quad\left(5.01 \times 10^{3}\right), \quad 688$ $\left(9.34 \times 10^{3}\right) \mathrm{nm} ;{ }^{1} \mathrm{H}$ NMR $\left(\mathrm{CDCl}_{3}, 400 \mathrm{MHz}\right) \delta:-0.90$ (br s, $1 \mathrm{H}, \mathrm{NH}),-0.95$ (br s, $1 \mathrm{H}, \mathrm{NH}), 1.54$ (t, $J=6.4 \mathrm{~Hz}$, $\left.3 \mathrm{H}, 8 \mathrm{a}-\mathrm{CH}_{3}\right), 1.70\left(\mathrm{~d}, J=6.9 \mathrm{~Hz}, 3 \mathrm{H}, 18-\mathrm{CH}_{3}\right), 1.90 \sim 2.03$ $(\mathrm{m}, 1 \mathrm{H}, \quad 17 \mathrm{a}+17 \mathrm{~b}-\mathrm{H}), 2.20 \sim 2.30(\mathrm{~m}, 2 \mathrm{H}, 17 \mathrm{a}+17 \mathrm{~b}-\mathrm{H})$, $2.40 \sim 2.51(\mathrm{~m}, 1 \mathrm{H}, 17 \mathrm{a}+17 \mathrm{~b}-\mathrm{H}), 2.88,2.93,3.35,3.67$ (each s, each $3 \mathrm{H}, \mathrm{CH}_{3}+\mathrm{OCH}_{3}$ ), 3.35 (q, $J=6.4 \mathrm{~Hz}, 2 \mathrm{H}$, $8 \mathrm{~b}-\mathrm{H}), 4.11$ (d, $J=10.4 \mathrm{~Hz}, 1 \mathrm{H}, 17-\mathrm{H}), 3.89$ (q, $J=6.4 \mathrm{~Hz}$, $1 \mathrm{H}, 18-\mathrm{H}), 6.14$ (d, $J=11.6 \mathrm{~Hz}, 1 \mathrm{H}$, cis-3b-H), 6.22 (d, $J=18.0 \mathrm{~Hz}, 1 \mathrm{H}$, trans-3b-H), 6.59 (br s, $1 \mathrm{H}, \mathrm{OH}), 7.36$ (s, $\left.1 \mathrm{H}, 13^{2}=\mathrm{CH}\right), 7.82(\mathrm{dd}, J=17.7,11.6 \mathrm{~Hz}, 3 \mathrm{a}-\mathrm{H}), 7.66$, $8.33,8.75$ (each s, each 1H, meso-H); IR (KBr) v: 33419 $(\mathrm{N}-\mathrm{H}), 2915,2847(\mathrm{C}-\mathrm{H}), 1733,1691(\mathrm{C}=\mathrm{O}), 1604$ $(\mathrm{C}=\mathrm{C}), 1497$ (chlorin skeleton), 1437, 1365, 1199, 1111, $981 \mathrm{~cm}^{-1}$; EI-MS m/z: $577.4\left(\mathrm{M}+\mathrm{H}^{+}\right)$. Anal. calcd for $\mathrm{C}_{35} \mathrm{H}_{36} \mathrm{~N}_{4} \mathrm{O}_{4}$ : C 72.90, H 6.29, N 9.72; found $\mathrm{C} 72.81, \mathrm{H}$ $6.09, \mathrm{~N} 9.59$.

\section{$3.7 N$-苯甲基红紫素-18 二亚酰胺甲酯(9)的合成}

将 $120 \mathrm{mg}$ 红紫素-18 甲酯 3 (0.207 mmol) 溶解于 15 $\mathrm{mL}$ 吡啶中, 再加入 $1 \mathrm{~mL}$ 芐胺, 室温搅拌反应 $5 \mathrm{~h}$ 后, 加 入 $220 \mathrm{~mL}$ 盐酸水溶液 $(200 \mathrm{~mL}$ 水 $+20 \mathrm{~mL}$ 浓盐酸), 并 用二氯甲烷萃取 $(80 \mathrm{~mL} \times 3)$, 合并有机相, 用水洗涤除 酸(100 $\mathrm{mL} \times 3)$, 经无水硫酸钠干燥和重氮甲烷甲基化, 减压浓缩, 剩余物用硅胶柱层析[洗脱剂: $V$ (石油醚) : $V($ 乙酸乙酯 $)=3: 1]$ 分离, 得 $82 \mathrm{mg}$ 紫红色固体 $9(0.122$ mmol ), 产率 59\%. m.p. 259 261 ${ }^{\circ} \mathrm{C}$; UV-Vis $\left(\mathrm{CHCl}_{3}\right)$

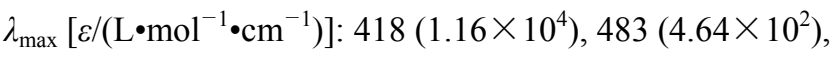
$511\left(5.81 \times 10^{2}\right), 550\left(1.97 \times 10^{3}\right), 650\left(6.96 \times 10^{2}\right), 706$ $\left(4.06 \times 10^{3}\right) \mathrm{nm} ;{ }^{1} \mathrm{H} \mathrm{NMR}\left(\mathrm{CDCl}_{3}, 400 \mathrm{MHz}\right) \delta:-0.16$ (br s, $1 \mathrm{H}, \mathrm{NH}),-0.07$ (br s, $1 \mathrm{H}, \mathrm{NH}), 1.64$ (t, $J=7.6 \mathrm{~Hz}$, 
$\left.3 \mathrm{H}, 8 \mathrm{a}-\mathrm{CH}_{3}\right), 1.75\left(\mathrm{~d}, J=7.3 \mathrm{~Hz}, 3 \mathrm{H}, 18-\mathrm{CH}_{3}\right), 1.94 \sim 2.05$ (m, 1H, 17a+17b-H), 2.32 2.46 (m, 2H, 17a+17b-H), $2.65 \sim 2.73(\mathrm{~m}, 1 \mathrm{H}, 17 \mathrm{a}+17 \mathrm{~b}-\mathrm{H}), 3.13,3.34,3.56,3.70$ (each s, each $3 \mathrm{H}, \mathrm{CH}_{3}+\mathrm{OCH}_{3}$ ), 3.63 (q, J=7.6 Hz, 2H, 8b-H), 4.34 (q, $J=7.3 \mathrm{~Hz}, 1 \mathrm{H}, 18-\mathrm{H}), 4.72$ (d, $J=14.4 \mathrm{~Hz}$, $2 \mathrm{H}, \mathrm{NCH}_{2} \mathrm{Ph}$ ), 5.38 (d, J=8.2 Hz, 1H, 17-H), 6.13 (dd, $J=11.6,1.0 \mathrm{~Hz}, 1 \mathrm{H}$, cis-3b-H), 6.27 (dd, $J=17.8,1.0 \mathrm{~Hz}$, 1H, trans-3b-H), 7.27 (d, J=7.4 Hz, 1H, Ph-H ), 7.38 (t, $J=7.7 \mathrm{~Hz}, 2 \mathrm{H}, \mathrm{Ph}-\mathrm{H}$ ), 7.75 (d, $J=7.4 \mathrm{~Hz}, 2 \mathrm{H}, \mathrm{Ph}-\mathrm{H})$, 7.87 (dd, $J=17.8,11.6 \mathrm{~Hz}, 1 \mathrm{H}, 3 \mathrm{a}-\mathrm{H}), 8.56,9.32,9.56$ (each s, each $1 \mathrm{H}$, meso-H); ${ }^{13} \mathrm{C}$ NMR $\left(400 \mathrm{~Hz}, \mathrm{CDCl}_{3}, 400\right.$ $\mathrm{MHz}) \delta$ : 11.99, 16.23, 16.49, 21.61, 22.02, 29.52, 29.70, $30.71,30.80,47.42,48.69,48.28,51.75,52.80,53.50$, 56.21 , 104.15, 107.44, 109.27, 109.67, 111.85, 114.08, $118.26,121.94,128.89,128.69,131.57,131.74,136.70$, $142.01,147.49,147.57,149.15,149.27,152.64,162.79$, 163.62, 164.98, 171.43, 173.01, 174.71; IR (KBr) v: 3435 $(\mathrm{N}-\mathrm{H}), 2924(\mathrm{C}-\mathrm{H}), 1726,1679(\mathrm{C}=\mathrm{O}), 1621(\mathrm{C}=\mathrm{C})$, 1585 (chlorin skeleton), 1373, 1013, $912 \mathrm{~cm}^{-1}$; EI-MS m/z: $688.2\left(\mathrm{M}+\mathrm{H}^{+}\right)$. Anal. calcd for $\mathrm{C}_{41} \mathrm{H}_{41} \mathrm{~N}_{5} \mathrm{O}_{4}: \mathrm{C} 73.74, \mathrm{H}$ 6.19, N 10.49; found C 73.60, H 6.30, N 10.61.

\section{$3.8 \mathrm{~N}$-苯甲基-3-(2,2-二甲氧基乙基)-3-去乙烯基红紫 素-18 二酰亚胺甲酯(10)的合成}

将 $148 \mathrm{mg}$ 化合物 $9(0.222 \mathrm{mmol})$ 溶解于 $5 \mathrm{~mL}$ 二氯 甲烷中, 再加入 $20 \mathrm{~mL}$ 甲醇, 在冰水浴和剧烈搅拌条件 下，一次性加入溶解于 $30 \mathrm{~mL}$ 甲醇的 $120 \mathrm{mg}$ 三水硝酸 铊 $(0.262 \mathrm{mmol})$, 继续搅拌反应 $45 \mathrm{~min}$ 后加入 $30 \mathrm{~mL}$ 由 亚硫酸氢钠饱和的甲醇溶液, 撤去冰水浴使反应体系的 温度升至室温, 再搅拌 $20 \mathrm{~min}$, 滴加 $2 \mathrm{~mL}$ 浓盐酸酸化 体系, 除去所生成的白色沉淀, 先后加入 $30 \mathrm{~mL}$ 水和 35 $\mathrm{mL}$ 二氯甲烷, 分出有机层, 水层用二氯甲烷萃取 $(20$ $\mathrm{mL} \times 2)$, 合并有机层并水洗两次, 用无水硫酸钠干燥, 减压浓缩, 剩余物经硅胶柱层析分离[洗脱剂: $V$ (石油 醚)： $V$ (乙酸乙酯 $)=4: 1$ ], 得到 $115 \mathrm{mg}$ 黑绿色固体 10 (0.158 mmol). 产率 71\%. m.p. 191 $194{ }^{\circ} \mathrm{C}$; UV-Vis $\left(\mathrm{CH}_{2} \mathrm{Cl}_{2}\right) \lambda_{\max }\left[\varepsilon /\left(\mathrm{L} \cdot \mathrm{mol}^{-1} \cdot \mathrm{cm}^{-1}\right)\right]: 386\left(1.26 \times 10^{5}\right), 416$ $\left(9.32 \times 10^{4}\right), \quad 514 \quad\left(1.51 \times 10^{4}\right), \quad 619 \quad\left(6.30 \times 10^{3}\right), \quad 668$ $\left(7.06 \times 10^{4}\right) ;{ }^{1} \mathrm{H}$ NMR $\left(\mathrm{CDCl}_{3}, 400 \mathrm{MHz}\right) \delta: 0.04$ (br s, $1 \mathrm{H}$, $\mathrm{NH}), 0.11$ (br s, $1 \mathrm{H}, \mathrm{NH}), 1.63$ (t, $J=7.6 \mathrm{~Hz}, 3 \mathrm{H}, 8-\mathrm{CH}_{3}$ ), $1.84\left(\mathrm{~d}, J=7.0 \mathrm{~Hz}, 3 \mathrm{H}, 18-\mathrm{CH}_{3}\right), 1.98 \sim 2.12(\mathrm{~m}, 1 \mathrm{H}, 17 \mathrm{a}$ $+17 \mathrm{~b}-\mathrm{H}), 2.36 \sim 2.47(\mathrm{~m}, 1 \mathrm{H}, 17 \mathrm{a}+17 \mathrm{~b}-\mathrm{H}), 2.62 \sim 2.77$ (m, $2 \mathrm{H}, 17 \mathrm{a}+17 \mathrm{~b}-\mathrm{H}), 3.55$ (q. $J=7.6 \mathrm{~Hz}, 2 \mathrm{H}, 8 \mathrm{a}-\mathrm{H}), 3.10$, $3.30,3.49\left(2 \mathrm{CH}_{3}\right), 3.60\left(2 \mathrm{CH}_{3}\right)$ (each s, each $3 \mathrm{H}, 18 \mathrm{H}, \mathrm{CH}_{3}$ $\left.+\mathrm{OCH}_{3}\right), 3.60 \sim 3.68(\mathrm{~m}, 1 \mathrm{H}, 3 \mathrm{a}-\mathrm{H}), 4.01(\mathrm{~d}, J=4.8 \mathrm{~Hz}$, $\left.2 \mathrm{H}, \mathrm{PhCH}_{2} \mathrm{~N}\right), 4.42$ (q, $\left.J=7.2 \mathrm{~Hz}, 1 \mathrm{H}, 18-\mathrm{H}\right), 4.97$ (t, $J=$
$5.2 \mathrm{~Hz}, 1 \mathrm{H}, 3 \mathrm{~b}-\mathrm{H}), 5.47$ (d, $J=8.4 \mathrm{~Hz}, 1 \mathrm{H}, 17-\mathrm{H}), 7.35 \sim$ 7.53 (m, 3H, Ph-H), 7.96 (d, J=7.6 Hz, 1H, Ph-H), 8.76 (d, $J=7.8 \mathrm{~Hz}, 1 \mathrm{H}, \mathrm{Ph}-\mathrm{H}$ ), 8.57, 9.23, 9.31 (each s, each 1H, meso-H); IR (KBr) v: $3452(\mathrm{~N}-\mathrm{H}), 2957(\mathrm{C}-\mathrm{H})$, 1732, $1726(\mathrm{C}=\mathrm{O}), 1608(\mathrm{C}=\mathrm{C}), 1520$ (chlorin skeleton), 1434, 1363, 1256, 1089, $908 \mathrm{~cm}^{-1}$; EI-MS m/z: 730.3 $\left(\mathrm{M}+\mathrm{H}^{+}\right)$. Anal. calcd for $\mathrm{C}_{43} \mathrm{H}_{47} \mathrm{~N}_{5} \mathrm{O}_{6}: \mathrm{C} 70.76, \mathrm{H} 6.49, \mathrm{~N}$ 9.60; found C 70.87, H 6.53, N 9.74.

\section{$3.9 \mathrm{~N}$-苯甲基-3-甲酰甲基-3-去乙烯基红紫素-18 二酰 亚胺甲酯(11)的合成}

将 $112 \mathrm{mg}$ 化合物 $\mathbf{1 0}$ (0.154 mmol)溶解于 $10 \mathrm{~mL}$ $80 \%$ 的甲酸水溶液中，室温搅拌 $24 \mathrm{~h}$, 加 $20 \mathrm{~mL}$ 水和 40 $\mathrm{mL}$ 二氯甲烷萃取, 分出水层, 用 $15 \mathrm{~mL}$ 饱和碳酸氢钠 溶液洗涤, 再水洗一次, 干燥浓缩后用硅胶柱层析分离 [洗脱剂: $V$ (石油醚) : $V$ (乙酸乙酯 $)=4 ： 1$ ], 得 $84 \mathrm{mg}$ 红 色固体 11 (0.123 mmol), 产率 80\%. m.p. 205 207 ${ }^{\circ} \mathrm{C}$; UV-vis $\left(\mathrm{CH}_{2} \mathrm{Cl}_{2}\right) \lambda_{\max }\left[\varepsilon /\left(\mathrm{L} \cdot \mathrm{mol}^{-1} \cdot \mathrm{cm}^{-1}\right)\right]$ : $420(9.46 \times$ $\left.10^{4}\right), 520\left(1.14 \times 10^{4}\right), 5.67\left(4.80 \times 10^{3}\right), 638\left(4.80 \times 10^{3}\right)$, $672\left(3.87 \times 10^{4}\right) \mathrm{nm} ;{ }^{1} \mathrm{H} \mathrm{NMR}\left(\mathrm{CDCl}_{3}, 400 \mathrm{MHz}\right) \delta$ : 0.23 (br s, 1H, NH), 0.16 (br s, $1 \mathrm{H}, \mathrm{NH}), 1.65$ (t, $J=7.6$ $\left.\mathrm{Hz}, 3 \mathrm{H}, 8-\mathrm{CH}_{3}\right), 1.76$ (d, $\left.J=7.2 \mathrm{~Hz}, 3 \mathrm{H}, 18-\mathrm{CH}_{3}\right), 1.91 \sim$ $2.04(\mathrm{~m}, 1 \mathrm{H}, 17 \mathrm{a}+17 \mathrm{~b}-\mathrm{H}), 2.28 \sim 2.43(\mathrm{~m}, 2 \mathrm{H}, 17 \mathrm{a}+$ $17 \mathrm{~b}-\mathrm{H}), 2.64 \sim 2.75(\mathrm{~m}, 1 \mathrm{H}, 17 \mathrm{a}+17 \mathrm{~b}-\mathrm{H}), 3.16,3.28,3.59$, 3.80 (each s, each $3 \mathrm{H}, \mathrm{CH}_{3}+\mathrm{OCH}_{3}$ ), 3.64 (q, $J=7.6 \mathrm{~Hz}$ 2H, 8a-H), 4.32 (q, $J=7.2 \mathrm{~Hz}, 1 \mathrm{H}, 18-\mathrm{H}$ ), 4.81 (br s, 2H, $3 \mathrm{a}-\mathrm{H}), 5.41$ (d, $J=8.8 \mathrm{~Hz} 1 \mathrm{H}, 17-\mathrm{H}), 5.67$ (d, $J=14.4 \mathrm{~Hz}$, $\left.1 \mathrm{H}, \mathrm{PhCH}_{2} \mathrm{~N}\right), 5.73$ (d, J=14.4 Hz, 1H, $\left.\mathrm{PhCH}_{2} \mathrm{~N}\right), 7.29$ (t, $J=7.6 \mathrm{~Hz}, 1 \mathrm{H}, \mathrm{Ph}-\mathrm{H}), 7.38$ (t, $J=7.6 \mathrm{~Hz}, 2 \mathrm{H}, \mathrm{Ph}-\mathrm{H}), 7.75$ (d, $J=7.6 \mathrm{~Hz}, 2 \mathrm{H}, \mathrm{Ph}-\mathrm{H}$ ), 9.15, 8.58, 8.09 (each s, each 1H, meso-H), 9.60 (s, 1H, CHO); IR (KBr) v: $3442(\mathrm{~N}-$ $\mathrm{H}), 2966(\mathrm{C}-\mathrm{H}), 1741,1735,1689(\mathrm{C}=\mathrm{O}), 1650(\mathrm{C}=\mathrm{C})$, 1563 (chlorin skeleton), 1438, 1401, 1256. 1166, 1081, 722 $\mathrm{cm}^{-1}$; EI-MS m/z: $684.4\left(\mathrm{M}+\mathrm{H}^{+}\right)$. Anal. calcd for $\mathrm{C}_{41} \mathrm{H}_{41} \mathrm{~N}_{5} \mathrm{O}_{5}$ : C 72.02, H 6.04, N 10.24; found $\mathrm{C} 72.20, \mathrm{H}$ 6.07, N 10.46 .

\subsection{3-(2,2-二甲氧基乙基)-3-去乙烯基焦脱镁叶绿} 酸-a 甲酯(12)和 12-二甲氧甲基焦脱镁叶绿酸-a 甲酯 (13)的合成

将 $432 \mathrm{mg}$ 化合物 1 (0.787 mmol)溶解于 $20 \mathrm{~mL}$ 二氯 甲烷中, 再加入 $100 \mathrm{~mL}$ 甲醇, 在冰水浴和剧烈搅拌条 件下，一次性加入溶解于 $120 \mathrm{~mL}$ 甲醇的 $525 \mathrm{mg}$ 三水硝 酸铊 $(1.181 \mathrm{mmol})$, 摚拌反应 $1 \mathrm{~h}$, 加入 $120 \mathrm{~mL}$ 由亚硫酸 氢钠饱和的甲醇溶液, 温度条件下再搅拌 $30 \mathrm{~min}$, 向反 应体系滴加 $15 \mathrm{~mL}$ 浓盐酸. 过滤除去白色沉淀, 再向滤 液加入 $120 \mathrm{~mL}$ 水和 $150 \mathrm{~mL}$ 二氯甲烷, 分出有机层, 水 
层用二氯甲烷萃取 $(40 \mathrm{~mL} \times 2)$. 合并有机层并水洗两次, 用无水硫酸钠干燥, 减压浓缩, 剩余物经硅胶柱层析分 离[洗脱剂: $V$ (石油醚) $: V$ (乙酸乙酯) $=4: 1$ ], 分别得到 $327 \mathrm{mg}$ 黑绿色固体 12 (0.535 mmol, 68\%)和 $16 \mathrm{mg}$ 绿色 固体 $13(0.016 \mathrm{mmol}, 3 \%)$. 化合物 $\mathbf{1 2}$ 的物理和分析数据 与文献值一致 ${ }^{[12]}$. 13: m.p. $191 \sim 194^{\circ} \mathrm{C}$; UV-Vis $\left(\mathrm{CH}_{2} \mathrm{Cl}_{2}\right)$

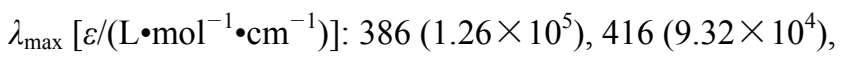
$514\left(1.51 \times 10^{4}\right), 619\left(6.30 \times 10^{3}\right), 668\left(7.06 \times 10^{4}\right) ;{ }^{1} \mathrm{H}$ NMR $\left(\mathrm{CDCl}_{3}, 400 \mathrm{MHz}\right) \delta$ : $-1.56(\mathrm{br} \mathrm{s}, 1 \mathrm{H}, \mathrm{NH}), 0.61$ (br s, $1 \mathrm{H}, \mathrm{NH}), 1.71$ (t, $\left.J=7.6 \mathrm{~Hz}, 3 \mathrm{H}, 8-\mathrm{CH}_{3}\right), 1.80$ (d, $\left.J=7.2 \mathrm{~Hz}, 3 \mathrm{H}, 18-\mathrm{CH}_{3}\right), 1.60 \sim 1.71(\mathrm{~m}, 1 \mathrm{H}, 17 \mathrm{a}+$ 17b-H), $1.76 \sim 1.90(\mathrm{~m}, 1 \mathrm{H}, \quad 17 \mathrm{a}+17 \mathrm{~b}-\mathrm{H}), 1.96 \sim 2.07$ (m, $1 \mathrm{H}, 17 \mathrm{a}+17 \mathrm{~b}-\mathrm{H}), 2.14 \sim 2.36(\mathrm{~m}, 1 \mathrm{H}, 17 \mathrm{a}+17 \mathrm{~b}-\mathrm{H})$, $3.29,3.38,3.47,3.61,3.91$ (each s, each $3 \mathrm{H}, 18 \mathrm{H}, \mathrm{CH}_{3}+$ $\mathrm{OCH}_{3}$ ), 3.77 (q, $J=7.6 \mathrm{~Hz}, 2 \mathrm{H}, 8 \mathrm{a}-\mathrm{H}$ ), 4.08 (dd, $J=9.2$, $2.4 \mathrm{~Hz}, 1 \mathrm{H}, 17-\mathrm{H}), 4.42(\mathrm{q}, \quad J=7.2 \mathrm{~Hz}, 1 \mathrm{H}, 18-\mathrm{H}), 5.97$ (d, $\left.J=6.0 \mathrm{~Hz}, 1 \mathrm{H}, 13^{2}-\mathrm{H}\right), 6.09$ (s, 1H, 12a-H), 6.15 (d, $\left.J=6.0 \mathrm{~Hz}, 1 \mathrm{H}, 13^{2}-\mathrm{H}\right), 6.25(\mathrm{~d}, J=11.2 \mathrm{~Hz}, 1 \mathrm{H}, c i s-3 \mathrm{~b}-\mathrm{H})$, 6.35 (d, $J=18.0 \mathrm{~Hz}, 1 \mathrm{H}$, trans-3b-H), 8.06 (dd, $J=18.0$, $11.2 \mathrm{~Hz}, 1 \mathrm{H}, 3 \mathrm{a}-\mathrm{H}$ ), 8.78, 9.60, 9.71 (each s, each $1 \mathrm{H}$, meso-H); IR (KBr) v: $3443(\mathrm{~N}-\mathrm{H}), 2832(\mathrm{C}-\mathrm{H}), 1736$, $1688(\mathrm{C}=\mathrm{O}), 1653(\mathrm{C}=\mathrm{C}), 1527$ (chlorin skeleton), 1400, 1287, 1189, $793 \mathrm{~cm}^{-1}$; EI-MS m/z: $609.4\left(\mathrm{M}+\mathrm{H}^{+}\right)$. Anal. calcd for $\mathrm{C}_{34} \mathrm{H}_{40} \mathrm{~N}_{4} \mathrm{O}_{5}$ : C 71.03, H 6.62, N 9.20; found $\mathrm{C}$ 71.09, H 6.50, N 9.31.

\section{$3.123 a$-甲酰基-3b-苯基焦脱镁叶绿酸-a 甲酯(15)的 合成}

取 $132 \mathrm{mg}$ 二氢卟吩二甲缩醛 $\mathbf{1 2}(0.216 \mathrm{mmol})$ 溶解 于 $10 \mathrm{~mL} 88 \%$ 的甲酸溶液中, 室温摚拌 $24 \mathrm{~h}$, 先后加 20 $\mathrm{mL}$ 水和 $40 \mathrm{~mL}$ 二氯甲烷, 分出有机层, 再分别用 $15 \mathrm{~mL}$ 饱和碳酸氢钠溶液和 $20 \mathrm{~mL}$ 水洗涤. 用无水硫酸钠干燥 后减压除尽溶剂. 将粗产物 14 溶解于溶有 $23 \mathrm{mg}$ 金属 钠的 $5 \mathrm{~mL}$ 甲醇中, 再加入 $66 \mathrm{mg}$ 苯甲醛 $(0.624 \mathrm{mmol})$, 氮气保护下于 $35{ }^{\circ} \mathrm{C}$ 恒温油浴摚拌反应 $6 \mathrm{~h}$, 先后加入 $25 \mathrm{~mL}$ 二氯甲烷和 $30 \mathrm{~mL}$ 水分层, 分出有机相, 水相用 二氯甲烷萃取 $(15 \mathrm{~mL} \times 2)$. 合并有机相, 再加入过量的 自制重氮甲烷乙梄溶液甲基化, 振摇 $2 \mathrm{~min}$ 后滴加 $1 \mathrm{~mL}$ 乙酸以除去剩余的重氮甲烷, 减压浓缩, 剩余物经硅胶 柱层析分离 [洗脱剂: $V$ (石油醚) $: V$ ( 乙酸乙酯 $)=4: 1$ ], 分别得到 $42 \mathrm{mg}$ 黑绿色固体 15 (0.065 mmol), 产率 30\%. m.p. $191 \sim 194^{\circ} \mathrm{C}$; UV-Vis $\left(\mathrm{CH}_{2} \mathrm{Cl}_{2}\right) \lambda_{\max }\left[\varepsilon /\left(\mathrm{L}^{\circ} \mathrm{mol}^{-1} \bullet\right.\right.$ $\left.\mathrm{cm}^{-1}\right)$ ]: $386\left(1.26 \times 10^{5}\right), 416\left(9.32 \times 10^{4}\right), 514(1.51 \times$ $\left.10^{4}\right), 619\left(6.30 \times 10^{3}\right), 668\left(7.06 \times 10^{4}\right) ;{ }^{1} \mathrm{H} \mathrm{NMR}\left(\mathrm{CDCl}_{3}\right.$, $400 \mathrm{MHz}) \delta:-1.68$ (br s, $1 \mathrm{H}, \mathrm{NH}), 0.35$ (br s, $1 \mathrm{H}, \mathrm{NH})$, $1.64\left(\mathrm{t}, J=7.6 \mathrm{~Hz}, 3 \mathrm{H}, 8 \mathrm{~b}-\mathrm{CH}_{3}\right), 1.87(1.90)(\mathrm{d}, J=7.2 \mathrm{~Hz}$, $\left.3 \mathrm{H}, 18-\mathrm{CH}_{3}\right), 2.20 \sim 2.37(\mathrm{~m}, 2 \mathrm{H}, 17 \mathrm{a}+17 \mathrm{~b}-\mathrm{H}), 2.51 \sim$ $2.74(\mathrm{~m}, 2 \mathrm{H}, 17 \mathrm{a}+17 \mathrm{~b}-\mathrm{H}), 3.02$ (3.01), 3.15 (3.14), 3.65 (3.59), 3.67 (each s, each $3 \mathrm{H}, \mathrm{CH}_{3}+\mathrm{OCH}_{3}$ ), 3.64 (q, $J=$ $7.6 \mathrm{~Hz}, 2 \mathrm{H}, 8 \mathrm{a}-\mathrm{H}), 4.36$ (d, $J=9.1 \mathrm{~Hz}, 1 \mathrm{H}, 17-\mathrm{H}), 4.51$ (q, $J=7.3 \mathrm{~Hz}, 1 \mathrm{H}, 18-\mathrm{H}), 5.15\left(\mathrm{~d}, J=19.8 \mathrm{~Hz}, 1 \mathrm{H}, 13^{2}-\mathrm{H}\right)$, $5.30\left(\mathrm{~d}, J=19.8 \mathrm{~Hz}, 1 \mathrm{H}, 13^{2}-\mathrm{H}\right), 6.94(\mathrm{t}, J=7.4 \mathrm{~Hz}, 2 \mathrm{H}$, Ph-H), 7.26 (d, $J=7.4$ Hz, 2H, Ph-H), 7.10 (t, $J=7.4 \mathrm{~Hz}$, $1 \mathrm{H}, \mathrm{Ph}-\mathrm{H}$ ), 8.29 (s, 1H, 3b-H), 8.63, 8.94, 9.50 (each s, each $1 \mathrm{H}$, meso-H), $10.33(10.32)(\mathrm{s}, 1 \mathrm{H}, 3 \mathrm{a}-\mathrm{CHO}) ;{ }^{13} \mathrm{C}$ NMR $\left(\mathrm{CDCl}_{3}, 400 \mathrm{MHz}\right) \delta: 10.96,11.27,12.11$ (89), 14.11, 16.90, 17.46, 19.36 (19.48), 23.27, 29.54 (30.01), 30.98 (31.02), 50.43, 50.65 (50.57), 51.62 (51.58), 55.31 (55.58), 56.04 (56.28), 60.64, 61.58, 92.54 (92.65), 95.93 (95.95), 104.24, 108.68 (108.87), 127.69, 131.49, 135.74 (135.80), 137.29, 137.35, 137.44, 137.47, 141.73, 141.78, $142.42,145.17$ (145.21), 149.06, 150.46, 155.65, 161.53, 169.52 (169.75), 172.21 (172.24), 173.64, 198.85, 203.09 (203.34); IR(KBr)v: IR (KBr) v: 3541, $3365(\mathrm{~N}-\mathrm{H}), 2927$, $2879(\mathrm{C}-\mathrm{H}), 1739,1699(\mathrm{C}=\mathrm{O}), 1604(\mathrm{C}=\mathrm{C}), 1519$ (chlorin skeleton), 1440, 1392, 1188, 1041, $748 \mathrm{~cm}^{-1}$; EI-MS $m / z$ : $653.3\left(\mathrm{M}+\mathrm{H}^{+}\right)$. Anal. calcd for $\mathrm{C}_{41} \mathrm{H}_{40} \mathrm{~N}_{4} \mathrm{O}_{4}$ : C 75.44, H 6.18, N 8.58; found C 75.35, H 6.09, N 8.69.

\subsection{3-(2,2-二甲氧基乙基)-3-去乙烯基- $13^{2}$-氧代焦脱 镁叶绿酸-a 甲酯(16)的合成}

取 $264 \mathrm{mg}$ 二氢卟吩缩醛 $\mathbf{1 2}(0.428 \mathrm{mmol})$ 溶于 25 $\mathrm{mL}$ 四氢呋喃中, 加入 $0.673 \mathrm{~g} \mathrm{LiOH}$ (溶解于 $6 \mathrm{~mL}$ 水中, 用 $15 \mathrm{~mL} \mathrm{MeOH}$ 稀释)的开放容器中, 避光室温剧烈摚 拌 $4 \mathrm{~h}$, 用 $\mathrm{AcOH}$ 调至酸性 $(\mathrm{pH}=3)$. 先后加入 $40 \mathrm{~mL}$ 二 氯甲烷和 $50 \mathrm{~mL}$ 水分层, 用二氯甲烷萃取 $(15 \mathrm{~mL} \times 3)$, 合并有机层, 用无水硫酸钠干燥, 减压蒸干, 再用 10 $\mathrm{mL}$ 二氯甲烷溶解, 加入略超计算量的重氮甲烷振摇 1 $\min$ 后, 用 $\mathrm{AcOH}$ 快速淬灭, 加 $25 \mathrm{~mL}$ 二氯甲烷, 水洗两 次, 用无水硫酸钠干燥, 减压浓缩, 剩余物用硅胶柱层 析分离 [洗脱剂: $V($ 正己烷) $: V$ (乙酸乙酯 $)=4: 1$ ]得 58 mg 黄色固体 16 (0.094 mmol, 产率 22\%. m.p. 191 $194{ }^{\circ} \mathrm{C}$; UV-Vis $\left(\mathrm{CH}_{2} \mathrm{Cl}_{2}\right) \lambda_{\max }\left[\varepsilon /\left(\mathrm{L} \cdot \mathrm{mol}^{-1} \cdot \mathrm{cm}^{-1}\right)\right]: 386$ $\left(1.26 \times 10^{5}\right), 416\left(9.32 \times 10^{4}\right), 514\left(1.51 \times 10^{4}\right), 619$ $\left(6.30 \times 10^{3}\right), 668\left(7.06 \times 10^{4}\right) ;{ }^{1} \mathrm{H} \mathrm{NMR}\left(\mathrm{CDCl}_{3}, 400 \mathrm{MHz}\right)$ $\delta:-2.54($ br s, $1 \mathrm{H}, \mathrm{NH}),-0.07$ (br s, $1 \mathrm{H}, \mathrm{NH}), 1.67$ (t, $\left.J=7.6 \mathrm{~Hz}, 3 \mathrm{H}, 8-\mathrm{CH}_{3}\right), 1.91$ (d, $J=7.6 \mathrm{~Hz}, 3 \mathrm{H}, 18-\mathrm{CH}_{3}$ ), $2.27 \sim 2.40(\mathrm{~m}, 2 \mathrm{H}, 17 \mathrm{a}+17 \mathrm{~b}-\mathrm{H}), 2.66 \sim 2.86(\mathrm{~m}, 2 \mathrm{H}$, $17 \mathrm{a}+17 \mathrm{~b}-\mathrm{H}$ ), 3.29, 3.45, 3.49, 3.60 (each s, each $3 \mathrm{H}, 18 \mathrm{H}$, $\left.\mathrm{CH}_{3}+\mathrm{OCH}_{3}\right), 4.18(\mathrm{~d}, J=5.3 \mathrm{~Hz}, 2 \mathrm{H}, 3 \mathrm{a}-\mathrm{H}), 4.96$ (q, $J=$ $7.2 \mathrm{~Hz}, 1 \mathrm{H}, 18-\mathrm{H}), 5.04$ (t, $J=5.3 \mathrm{~Hz}, 1 \mathrm{H}, 3 \mathrm{~b}-\mathrm{H}), 5.17$ (d, $J=6.4 \mathrm{~Hz}, 1 \mathrm{H}, 17-\mathrm{H}$ ), 8.97, 9.54, 9.69 (each s, each $1 \mathrm{H}$, 
meso-H), IR (KBr) v: $3459(\mathrm{~N}-\mathrm{H}), 2959(\mathrm{C}-\mathrm{H}), 1737$, $1717(\mathrm{C}=\mathrm{O}), 1662(\mathrm{C}=\mathrm{C}), 1564$ (chlorin skeleton), 1443, 1241. 1171, 1074, $906 \mathrm{~cm}^{-1}$; EI-MS $m / z: 625.1\left(\mathrm{M}+\mathrm{H}^{+}\right)$. Anal. calcd for $\mathrm{C}_{36} \mathrm{H}_{40} \mathrm{~N}_{4} \mathrm{O}_{6}$ : C 69.21, H 6.45, N 8.97; found C 69.10, H 6.29, N 9.12.

\subsection{3-(3-喹啉基)-3-去乙烯基(2,3-n)-喹喔啉并焦脱 镁叶绿酸-a 甲酯(17)的合成}

将 $85 \mathrm{mg}$ 化合物 $\mathbf{1 6}(0.1361 \mathrm{mmol})$ 溶解于 $20 \mathrm{~mL}$ 干 燥的吡啶中, 加入 $0.1 \mathrm{~mL}$ 三氟乙酸和 $60 \mathrm{mg}$ 邻苯二胺 (0.555 mmol), 通过插入反应瓶底部的玻管通入氮气 10 min, 在氮气保护下室温搅拌 $2.5 \mathrm{~h}$, 减压除尽吡啶; 向 滤液加入 $10 \mathrm{~mL} 80 \%$ 的甲酸溶液, 室温摚拌 $24 \mathrm{~h}$. 再加 $10 \mathrm{~mL}$ 水和 $20 \mathrm{~mL}$ 二氯甲烷, 分出水层, 有机层分别用 $15 \mathrm{~mL}$ 饱和碳酸氢钠溶液和水洗涤, 减压除尽溶剂. 所 得混合物重新溶解于 $10 \mathrm{~mL}$ 含有 $0.1 \mathrm{~mL}$ 三氟乙酸和 68 $\mathrm{mg}$ 邻氨基苯甲醛的干燥吡啶中, 氮气保护下室温搅拌 $2 \mathrm{~d}$, 加入 $20 \mathrm{~mL}$ 水洗涤, 减压除尽溶剂, 剩余物经硅胶 柱层析分离[洗脱剂: $V$ (石油醚) $: V$ (乙酸乙酯 $)=4: 1$ ], 得 $24 \mathrm{mg}$ 紫红色固体 17 (0.033 mmol), 产率为 24\%. m.p.

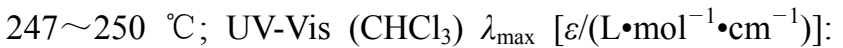
$372\left(4.89 \times 10^{4}\right), 424 \quad\left(1.02 \times 10^{5}\right), 480\left(7.14 \times 10^{3}\right), 556$ $\left(3.26 \times 10^{4}\right), 672\left(1.42 \times 10^{3}\right), 738\left(5.92 \times 10^{4}\right) \mathrm{nm} ;{ }^{1} \mathrm{H}$ NMR (400 MHz, $\left.\mathrm{CDCl}_{3}, 400 \mathrm{MHz}\right) \delta:-1.02($ br s, $1 \mathrm{H}$, $\mathrm{NH}),-0.50(\mathrm{br} \mathrm{s}, 1 \mathrm{H}, \mathrm{NH}), 1.51(\mathrm{t}, J=7.6 \mathrm{~Hz}, 3 \mathrm{H}$, $\left.8 \mathrm{~b}-\mathrm{CH}_{3}\right), 1.82\left(\mathrm{~d}, J=7.2 \mathrm{~Hz}, 3 \mathrm{H}, 18-\mathrm{CH}_{3}\right), 2.15 \sim 2.26(\mathrm{~m}$, $1 \mathrm{H}, 17 \mathrm{a}+17 \mathrm{~b}-\mathrm{H}), 2.29 \sim 2.38(\mathrm{~m}, 1 \mathrm{H}, 17 \mathrm{a}+17 \mathrm{~b}-\mathrm{H})$, $2.50 \sim 2.85(\mathrm{~m}, 2 \mathrm{H}, 17 \mathrm{a}+17 \mathrm{~b}-\mathrm{H}), 2.98,3.23,3.52,3.54$ (each s, each $3 \mathrm{H}, \mathrm{CH}_{3}+\mathrm{OCH}_{3}$ ), $3.46(\mathrm{q}, J=7.6 \mathrm{~Hz}, 2 \mathrm{H}$, $8 \mathrm{a}-\mathrm{H}), 4.09$ (q, $J=7.2 \mathrm{~Hz}, 1 \mathrm{H}, 18-\mathrm{H}), 5.22(\mathrm{~d}, J=8.4 \mathrm{~Hz}$, 1H, 17-H), 7.46 7.60 (m, 5H, Ar-H). 7.76 (br s, 1H, Ar-H), 7.85 (br s, 1H, Ar-H), 7.98 (dd, $J=7.2,4.0 \mathrm{~Hz}, 1 \mathrm{H}$, Ar-H), 8.17 (br s, 1H, Ar-H), 8.77 8.79 (m, 1H, Ar-H), 8.37, 8.93, 9.62 (each s, each $1 \mathrm{H}$, meso-H); IR (KBr) v: $3448(\mathrm{~N}-\mathrm{H}), 2926(\mathrm{C}-\mathrm{H}), 1739,1690(\mathrm{C}=\mathrm{O}), 1655$ $(\mathrm{C}=\mathrm{C}), 1551$ (chlorin skeleton), 1080, $780 \mathrm{~cm}^{-1}$; EI-MS $m / z: 736.2\left(\mathrm{M}+\mathrm{H}^{+}\right)$. Anal. calcd for $\mathrm{C}_{47} \mathrm{H}_{41} \mathrm{~N}_{7} \mathrm{O}_{2}: \mathrm{C}$ 76.71, H 5.62, N 13.32; found C 76.59, H 5.51, N 13.55.

辅助材料(Supporting Information) 所有新化合物的 核磁共振氢谱数据. 这些材料可以免费从本刊网站 (http://sioc-journal.cn/)上下载.

\section{References}

[1] (a) Liu, C.; Dobhal, M. P.; Ethirajan, M.; Missert, J. R.; Pandey, R. K.; Balasubramanian, S.; Sukumaran, D. K.; Zhang, M.; Kadish, K. M.; Ohkubo, K.; Fukuzumi, S. J. Am. Chem. Soc. 2008, 130, 14311. (b) Machida, S.; Isoda, Y.; Kunieda, M.; Tamiaki, H. Tetrahedron Lett. 2012, 53, 6277 .

(c) Wang, L.-M.; Wang, P.; Liu, C.; Jin, Y.-X.; Wang, J.-J. Chin. J. Org. Chem. 2012, 32, 1700 (in Chinese).

(王鲁敏，王朋，刘超，金英学，王进军，有机化学，2012，32， 1700.)

(d) Li, J.-Z.; Liu, W.-H.; Li, F.-G.; Wang, J.-J.; Suo, Y.-R.; Liu, Y.-J. Chin. J. Org. Chem. 2007, 27, 1594 (in Chinese).

(李家柱, 刘万卉, 李付国, 王进军, 索有瑞, 刘永军, 有机化学, 2007, 27, 1594.)

[2] (a) Chen, Y. H.; Li, G. L.; Pandey, R. K. Curr. Org. Chem. 2004, 8, 1105.

(b) Wang, J.-J. Chin. J. Org. Chem. 2005, 25, 1353 (in Chinese). (王进军, 有机化学, 2005, 25, 1353.)

(c) Yang, Z.; Wang, Z.; Liu, Y.; Xu, X.-S.; Qi, C.-X.; Wang, J.-J. Chin. J. Org. Chem. 2013, 33, 116 ( in Chinese).

(杨泽, 王振, 刘洋, 徐希森, 祁彩霞, 王进军, 有机化学, 2013, 33, 116.)

[3] (a) Ethirajan, M.; Joshi, P.; William, W. H.; Ohkubo, K.; Fukuzumi, S.; Pandey, R. K. Org. Lett. 2011, 13, 1956.

(b) Pavlov, V. Y.; Ponomarev, G. V. Chem. Heterocycl. Compd. 2004, 40, 393.

[4] (a) Xu, X.-S.; Yao, N.-N.; Liu, Y.; Yin, J.-G.; Qi, C.-X.; Wang, J.-J. Chin. J. Org. Chem, 2014, 34, 938 ( in Chinese).

(徐希森, 姚楠楠, 刘洋, 殷军港, 祁彩霞, 王进军, 有机化学, 2014, 34, 938.)

(b) Liu, Y.; Xu, X.-S.; Li, J.-Z.; Yin, J.-G.; Qi, C.-X.; Wang, J.-J. Chin. J. Org. Chem, 2014, 34, 552 (in Chinese).

(刘洋, 徐希森, 李家柱, 殷军港, 祁彩霞, 王进军, 有机化学, 2014, 34, 552.)

[5] (a) Wang, J.-J.; Wang, P.; Li, J.-Z.; Jakus, J.; Shin, Y.-K. Bull. Korean Chem. Soc. 2011, 32, 3473.

(b) Wang, J.-J.; Yin, Y.-F.; Yang, Z. J. Iran. Chem. Soc. 2013, 10, 583.

(c) Wang, J-J.; Ji, J.-Y.; Han, G. F.; Wu, X.-R.; Wang, L.-M.; Shin, Y.-K. Acta Chim. Sinica 2004, 62, 302 (in Chinese).

(王进军, 纪建业, 韩光范, 邬旭然, 王鲁敏, 沈荣基, 化学学报, 2004, 62, 302.)

[6] (a) Li, J.-Z.; Zhang, P.; Yao, N.-N.; Zhao, L.-L.; Wang, J.-J.; Shim, Y.-K. Tetrahedron Lett. 2014, 55, 1086.

(b) Ji, J.-Y.; Wang, L. M.; Jing, J.-R.; Han, G.-F.; Wang, J. J. Chin. J. Org. Chem. 2007, 27, 493 (in Chinese).

(纪建业, 王鲁民, 荆济荣, 韩光范, 王进军, 有机化学, 2007, 27, 493.)

(c) Wang, J.-J.; Li, J.-Z.; Li, Y.-W.; Jakus, J.; Shim, Y.-K. J. Porphyrins Phthalocyanines 2010, 14, 859.

[7] Wu, J. M.S. Thesis, Yantai University, Yantai, 2012 (in Chinese). (武进, 硕士论文, 烟台大学, 烟台, 2012.)

[8] Liu, Y. M.S. Thesis, Yantai University, Yantai, 2014 (in Chinese). (刘洋，硕士论文，烟台大学，烟台，2014.)

[9] (a) Ji, J.-Y.; Wang, L. M.; Jing, J.-R.; Han, G.-F.; Wang, J. J. Chin. J. Org. Chem. 2007, 27, 493 (in Chinese).

(纪建业，王鲁民，荆济荣，韩光范，王进军，有机化学，2007, 27, 493.)

(b) Srivatsan, A.; Wang, Y.-F.; Joshi, P.; Sajjad, M.; Chen, Y.-H.; Liu, C.; Thankcppan, K.; Missert, J. R.; Tracy, E.; Morgan, J.; Rigual, N.; Baumann, H.; Pandey, R. K. J. Med. Chem. 2011, 54, 6859.

[10] (a) Yu, S.-S.; Xu, X.-S.; Liu, Y.; Li, J.-Z.; Jin, Y.-X.; Qi, C.-X.; Wang, J.-J. Chin. J. Org. Chem. 2014, 34, 362 (in Chinese). (于沙沙, 徐希森, 刘洋, 李家柱, 金英学, 祁彩霞, 王进军, 有 机化学, 2014, 34, 362.) 
(b) Tamiaki, H.; Monobe, R.; Koizumi, S.; Miyatake, T.; Kinoshita, Y. Tetrahedron: Asymmerty 2013, 24, 966.

(c) Yin, Y.-F.; Zhang, Q.; Liu, Y.; Xu, X.-S.; Qi, C.-X.; Wang, J.-J. Chin. J. Org. Chem. 2013, 33, 581 (in Chinese).

(殷一柇, 张千, 刘洋, 徐希森, 祁彩霞, 王进军, 有机化学, 2013, 33, 581.)
[11] Smith, K. M.; Gogg, D. A.; Simpson, D. J. J. Am. Chem. Soc. 1985, $107,4946$.

[12] Wang, J.-J.; Han, G.-F.; Yin, J.-G.; Wu, X.-R.; Zhao, Y.; Gong, B.-A.; Shin, R.-K. Acta Chim. Sinica 2003, 61,97 (in Chinese). (王进军, 韩光范, 殷军港, 邬旭然, 赵岩, 宫宝安, 沈荣基, 化 学学报, 2003, 61, 97.)

(Li, L.; Fan, Y.) 